\title{
La prima traduzione francese della "Pamela" di Richardson
}

\section{Franco Piva}

\section{(2) OpenEdition}

1 Journals

\section{Edizione digitale}

URL: http://journals.openedition.org/studifrancesi/5489

DOI: 10.4000/studifrancesi.5489

ISSN: 2421-5856

\section{Editore}

Rosenberg \& Sellier

\section{Edizione cartacea}

Data di pubblicazione: 1 septembre 2011

Paginazione: 255-280

ISSN: 0039-2944

\section{Notizia bibliografica digitale}

Franco Piva, «La prima traduzione francese della "Pamela" di Richardson», Studi Francesi [Online], 164 (LV | II) | 2011, online dal 30 novembre 2015, consultato il 07 janvier 2021. URL: http:// journals.openedition.org/studifrancesi/5489 ; DOI: https://doi.org/10.4000/studifrancesi.5489

\section{cc)}

Studi Francesi è distribuita con Licenza Creative Commons Attribuzione - Non commerciale - Non opere derivate 4.0 Internazionale. 


\section{La prima traduzione francese della "Pamela" di Richardson}

Apparsa in libreria verso la fine del $1740^{1}$, anche se con la data del 1741, $\mathrm{Pa}$ mela, or Virtue rewarded, il romanzo che doveva trasformare il tipografo Samuel Richardson in uno scrittore subito celebre, conobbe, come è noto, un successo tanto inatteso quanto sfolgorante: una seconda edizione vide infatti la luce agli inizi di febbraio del 1741, una terza a marzo ed una quarta a maggio, mentre la quinta sarebbe uscita, di lì a pochi mesi, in ottobre ${ }^{2}$. Come ha fatto osservare uno dei più noti studiosi di Richardson, «It is safe to say that almost everybody read it [vale a dire Pamela]»; al punto che il romanzo divenne ben presto «the fashion, the best seller» che non solo era necessario leggere, ma che non mancò neppure, con la sua straordinaria novità, di suscitare una vera e propria querelle che vide per mesi su fronti opposti i più fanatici ammiratori di Richardson fieramente schierati contro coloro che, altrettanto violentemente, a lui ed alla sua opera si opposero, per ragioni peraltro più morali che prettamente letterarie, oltre ad una nutrita serie di adattamenti, imitazioni e parodie ${ }^{3}$.

$\grave{E}$ in questo contesto che, a neppure un anno di distanza dalla pubblicazione, prese corpo e fu realizzata la prima traduzione francese della Pamela di Richardson. La cosa potrebbe sembrare, ed in qualche modo in effetti è logica, visto che il francese era la lingua tramite la quale la maggior parte dei lettori dell'epoca entravano in contatto con tutto ciò che di interessante veniva allora scritto e stampato in Europa, e visto l'interesse che la Francia da qualche tempo nutriva nei riguardi dell'Inghilterra, anche se gli anni che videro la prima traduzione francese di Pamela si situano solo all'inizio di quel vasto e complesso fenomeno dell' anglomania che, nella seconda parte del Settecento, avrebbe spinto i Francesi non solo ad interessarsi a tutto ciò che si faceva e si scriveva al di là della Manica, ma a mostrare per quanto proveniva dall'Inghilterra un interesse in qualche modo fanatico, una «mania», per l'appunto ${ }^{4}$. La prima traduzione francese di Pamela avvenne tuttavia in circostanze e secondo modalità che gli studiosi che si sono interessati a Richardson ed alla sua opera non sono ancora riusciti a chiarire in maniera soddisfacente, al punto che su tutta la complessa vicenda permangono delle ampie zone d'ombra e non pochi punti ancora oscuri. Può essere quindi utile fare il punto sulla situazione e cercare di apportare sulla delicata questione, per quanto è possibile e per quanto lo consentano $i$ documenti di cui disponiamo, qualche ulteriore chiarimento.

(1) Esattamente il 6 novembre 1740 (cfr. il Daily Gazetteer dello stesso giorno, citato da W. M. SALE, Samuel Richardson. A Bibliographical Record of the literary Career with historical notes, New Haven, Yale University Press, 1932, p 14.)

(2) Cfr W. M. SAlE, Samuel Richardson. A Bibliographical Record cit., pp. XV-XVI e A. D. McKILLOP, Samuel Richardson Printer and Novelist, Chapel Hill, The University of North Carolina Press, 1936, p. 43.
(3) I materiali relativi a questa querelle sono stati raccolti, alcuni anni orsono, in sei volumi da Thomas Kreymer e Peter Sabor. Cfr. The "Pamela" controversy: criticism and adaptations of Samuel Richardson's "Pamela" 1740-1750. Edited by Thomas Kreymer and Peter SABOR, London, Pickering and Chatto, 2001.

(4) Cfz. J. Grieder, Anglomania in France. 1740 1789. Fact, fiction and political discourse, Genève, Librairie Droz, 1985. 


\section{La traduzione}

Sarebbe stato logico pensare che l'idea di tradurre un'opera che nel suo paese aveva conosciuto un successo tanto strepitoso quanto inatteso fosse venuta a qualche editore francese, pronto ad approfittare della favorevole occasione. Pare invece ormai assodato che l'operazione sia nata e sia stata realizzata a Londra: non soltanto i due tomi di Pamela; ou la Vertu Recompensée. Traduit de l'Anglois apparvero «A Londres, chez Thomas Woodward, au Croissant entre les Portes du Temple; et Jean Osborn, à la Boucle d'Or, dans Pater Noster Row, près de S. Paul» con la data del 1741, - indicazione importante, anche se di per sé non indiscutibile in quanto gli editori francesi ricorrevano spesso, come è noto, per ragioni legate al privilège o alla censura, ad adresses straniere - ma tutte le indicazioni al riguardo di cui attualmente disponiamo conducono in quella direzione. Così è per la Bibliothèque britannique che in un articolo su Pamela, or Virtue rewarded, datato febbraio 1741, dopo aver ricordato il grande successo che il romanzo di Richardson aveva subito incontrato in Inghilterra, annunciava che «on le traduit actuellement en François» assicurando che «la Traduction est déjà si avancée, qu'elle paroîtra peut-être aussi-tôt que cet extrait», uscito esso nel tomo XVII. Première Partie della Bibliothèque «pour les mois d'Avril, Mai \& Juin M.DCC.XLI» ${ }^{5}$; così è per il Daily Post che nel numero del 27 marzo 1741 annunciava che la traduzione francese di Pamela era «in the Press» e che sarebbe stata «speedly [...] published $»^{6}$. Sappiamo d'altra parte che almeno il primo volume della traduzione francese è uscito dai torchi dallo stampatore londinese William Bowyer ${ }^{7}$, anziché da quelli dello stesso Richardson, come ci si sarebbe potuti aspettare, forse perché, come hanno suggerito gli editori della "Pamela" Controversy, Bowyer disponeva di «compositors with competence in French».

Da chi fu presa l'iniziativa di tradurre il romanzo di Richardson in Inghilterra, e per quale motivo, con quali fini? Paul Dottin ha scritto, all'inizio del secolo scorso, che la decisione fu presa dallo stesso Richardson: da un lato per rispondere alle richieste sempre più pressanti che provenivano dal Continente ( «Il ne se passait pas de semaine, que des amis n'apportassent à l'heureux père de Pamela de bonnes nouvelles des pays conquis par sa fille»), dall'altro per anticipare eventuali probabili profittatori («Richardson, sachant que le Copyright Act de 1710 ne protégeait l'œuvre littéraire qu'en Grande Bretagne, se préoccupa de trouver un traducteur habile et expéditif qui fût capable de préparer une Pamela française avant que les pirates eussent pu s'emparer de l'œuvre») e che per questo si rivolse all' «actif agent littéraire Fréval» il quale «pour aller vite, recruta toute une équipe de traducteurs qui s'attela à la traduction du roman»". Paul Dottin, che non cita quasi mai le sue fonti, non è uno studioso su cui si possa fare cieco affidamento: accanto ad indicazioni utili ed affidabili, se ne incontrano infatti altre che hanno tutta l'aria di essere di più o meno pura invenzione. Quella, appena citata, relativa alla traduzione di Pamela sembra appartenere alla seconda categoria. Essa è infatti contraddetta da un'altra, essa del tutto affidabile

(5) Cfr. Bibliothèque britannique, ou Histoire des ouvres des sçavans de la Grande Bretagne pour les mois d'Avril, Mai \& Juin M.DCC.XLI. Tome dixseptième. Première Partie. A la Haye, chez Pierre De Hondt, MDCCXLI, p. 60.

(6) Cfr. A. D. McKillop, Samuel Richardson cit., p. 92 e The "Pamela" Controversy cit., vol. II, p. XXIII.

(7) J. Nichols, Biographical and literary Anecdotes of William Bowyer, Bristol, Thoemmes Press; Tokio, Linokumya, 1996 (Fac-sim. de l'édition de
Londres, J. Nichols, 1782), t. III., p. 306 e The Bowyer Ledgers. The printing accounts of William Bow yer, father and son [...] Edited by Keits MASLEN and John LANCASTER, London, Bibliographical Society, 1991, item 2963.

(8) The "Pamela" Controversy cit., t. II, p. XXIII.

(9) P. DotTin, Samuel Richardson 1689-1764 Imprimeur de Londres, auteur de Pamela, Clarisse et Grandison, Paris, Librairie académique Perrin et Cie, Libraires- Editeurs, 1941, p. 117. 
e contemporanea ai fatti di cui stiamo parlando. Il Daily Gazetteer del 23 ottobre 1741, annunciando l'uscita della traduzione francese di Pamela, precisava che essa era stata fatta «for the Use of SCHOOLS ${ }^{10}$. Un'indicazione praticamente identica è stata trovata da Louis Thevenon nel Daily Advertiser dello stesso giorno: «This day is publis'd// For use in Schools// Pamela: ou la Virtue (sic!) récompensée//traduit de l'Anglois//En deux tomes// A Londres, chez Thomas Woodward, Au Croissant entre les Portes du Temple; et Jean Osborn à la Boule d'Or dans Pater-Noster Row, près de $\mathrm{S}$. Paul» ${ }^{11}$. La testimonianza offerta dai due giornali inglesi è importante; non solo perché ci dice che l'iniziativa di tradurre il romanzo non partì da Richardson, ma anche perché ci fa sapere che essa non nacque neppure dalla necessità di rispondere alle sempre più pressanti richieste che provenivano dal Continente, come aveva ipotizzato Dottin ${ }^{12}$, bensì da prospettive e con obiettivi diciamo così più modesti: mettere a disposizione delle scuole francesi, assai numerose a Londra, e più in generale in Inghilterra, dove il Rifugio era stato ed era ancora importante, un'opera di cui, come è noto, «Mr. le Docteur Slocok, Ministre de St. Sauveur dans le Southwak» aveva esaltato la moralità e l'utilità educativa al punto di raccomandarne «publiquement en Chaire la lecture» ${ }^{13}$.

Sebbene sia la Bibliothèque britannique che il Daily Post l'avessero data per imminente ancor prima della metà del 1741, e che una nota della Bibliotbèque Britannique redatta con ogni verosimiglianza ai primi di giugno, assicurasse che «la Traduction Françoise [...] qu'on imprime actuellement» sarebbe comparsa «dans peu de jours $\gg^{14}$, essa non comparve di fatto che quasi un anno dopo l'uscita dell'originale inglese: esattamente il 23 ottobre, come fu annunciato dal Daily Gazetteer e dal Daily Advertiser quello stesso giorno e dall'Evening Post nel numero del 24-27 ottobre ${ }^{15}$, quando Richardson stava già lavorando alla sesta edizione inglese della sua opera. C'erano stati chiaramente dei ritardi causati forse più dagli editori che dal o dai traduttori dell'opera.

Della traduzione francese di Pamela uscirono infatti in quel giorno, non una ma due edizioni: una che sulla page de titre del primo volume portava l'adresse: «A Londres, Chez Thomas Woodward, au Croissant entre les Portes du Temple; Et Jean Osborn, à la Boule d'Or, dans Pater Noster Row, près S. Paul»; ed un'altra che invece sulle pages de titre dei due volumi recava l'adresse del solo Jean Osborn, la stessa adresse che figurava sulla page de titre del secondo tomo dell'edizione precedente, che presentava anche un fleuron diverso da quello del primo tomo. A parte i due punti invece del punto e virgola dopo la Pamela del titolo (Pamela; ou la Vertu Recompensée nel primo caso; Pamela: ou la Vertu Recompensée nell'altro), l'assenza dell'indicazione «En deux Tomes» dopo il titolo e una maniera leggermente diversa di indicare l'indirizzo della Libreria («dans Pater-Noster-Row» anziché «dans Pater Noster Row») nella page di titre della prima rispetto alla seconda, le due edizioni del 1741 che abbiamo esaminato, e che sono conservate l'una alla British Library di Londra, l'altra alla Bodleian Library di Oxford ${ }^{16}$, non presentano tra di loro alcuna

(10) Cit. tratta da The "Pamela" Controversy cit. t. I, p. XXV. La stessa indicazione aveva dato W. M. SALE in Samuel Richardson. A Bibliographical Record cit., p. 16.

(11) L. THEVEnON, Étude de la sensibilité dans la traduction française de "Pamela" de Richardson, Thèse dactylographiée présentée à l'Université de Grenoble le 30 septembre 1977, p. 43.

(12) Le prime allusioni alla Pamela di Richardson non si incontrano, del resto, in Francia prima del- la metà del 1742, data alla quale la sua traduzione francese arrivò effettivamente nelle librerie di $\mathrm{Pa}$ rigi.

(13) Cfr. Bibliothèque britannique cit., t. XVII. Seconde Partie, p. 222.

(14) Ibid.

(15) Cfr. McKillop, Samuel Richardson cit., p. 93.

(16) Dove portano rispettivamente le segnature $1156 \mathrm{k}$ q e Vet A 4 f 464. 
differenza né nell'impaginazione né negli ornamenti che ne abbelliscono le pagine ${ }^{17}$; con una sola eccezione, vistosa però ed importante: l'edizione conservata alla British Library comporta, prima della «Préface de l'Editeur», una «Préface», che non reca alcuna qualificazione ma che potrebbe intitolarsi «Préface des Traducteurs», che non si trova invece nell'esemplare conservato alla Bodleiana di Oxford. Dopo aver ricordato lo straordinario successo incontrato dalla Pamela richardsoniana in Inghilterra $\mathrm{ma}$ anche le critiche che essa aveva via via suscitato, i Traduttori ${ }^{18}$ parlano della traduzione, a proposito della quale fanno presente che essi hanno cercato di «la rendre aussi fidelle qu'il [leur] a été possible vû la différence des Langues», precisando che poiché «la Langue Angloise n'est pas tout à fait aussi châtiée que la Françoise», essi sono stati non di rado costretti «à rendre le sens de notre Auteur plutôt que de suivre exactement ses Expressions» ${ }^{19}$. Proseguono poi formulando l'auspicio che «les sentimens d'Humanité, de Vertu, \& de Religion, \& la Varieté des Caractères justes \& touchés dont l'Original de cet Ouvrage est rempli, \& qui l'ont fait recevoir si favorablement des Anglois, seront cause que les Etrangers ne liront pas avec moins de plaisir la Traduction que nous leur présentons $\aleph^{20}$, con una lettura dell'opera perfettamente in linea con gli scopi che, stando alle indicazioni sopra riportate, $i$ traduttori si erano proposti, ma aperta anche ad un lettorato più ampio, come se, nel momento in cui si accinsero a tradurre Pamela, i traduttori non avessero presenti solo i destinatari primi del loro lavoro (gli studenti delle scuole francesi) ma anche altri e più numerosi lettori che il grande successo che l'opera stava incontrando in Inghilterra non avrebbe mancato di portare all'opera di Richardson anche sul Continente. E proprio in questa duplice prospettiva sta, forse, la ragione più probabile sia del ritardo con il quale la traduzione uscì rispetto alle previsioni, sia della concomitante doppia edizione. Nata inizialmente con ambizioni piuttosto modeste, indicate in quel «For the Use of Schools» che abbiamo dianzi ricordato, sotto l'egida dei due editori che compaiono sulla page de titre del primo tomo dell'esemplare conservato ad Oxford, il successo, certamente inatteso, che l'opera aveva incontrato nel frattempo indusse probabilmente gli editori, o almeno uno di essi, ad orientare la traduzione verso un altro lettorato e ad agire di conseguenza. Woodward, non condividendo l'idea o forse spaventato dall'impegno finanziario che l'operazione avrebbe comportato, si è probabilmente ritirato; a meno che non sia stato John Osborn che, avendo fiutato l'affare, abbia liquidato il socio, assumendo su di sé tutto l'onere ma anche i probabili profitti che un'operazione del genere non avrebbe mancato di assicurargli. È forse anche utile ricordare che John Osborn era stato co-editore, assieme a Charles Rivington, del testo originale; non è infatti da escludere che anche questa circostanza possa aver influito sulla risoluzione dell'accordo primitivamente stipulato tra John Osborn e Thomas Woodward

$\mathrm{La}$ «Préface» conteneva anche un'altra notizia importante: vale a dire che la traduzione francese di Pamela, iniziata verosimilmente dopo l'uscita della seconda edizione inglese dell'opera, di cui però i Traduttori non sembrano aver tenuto molto conto, era stata «faite avec la participation de l'Auteur, qui a eu la bonté - precisavano i Traduttori - de nous fournir un petit nombre d'Additions \& de Corrections.

(17) E che non sono, comunque, le gravures, a firma Punt, che aveva creduto di vedervi Paul Dottin, sbagliandosi con l'edizione uscita ad Amsterdam, «aux dépens des Libraires associés», nel 1744.

(18) Diciamo «traduttori» e non «traduttore» perché chi scrive usa un plurale che non sembra affatto majestatis. Sulla questione cfr., ad ogni modo, oltre, il paragrafo dedicato al Traduttore.
(19) Pamela; ou la Vertu Recompensée. Traduit de l'Anglois. En deux Tomes. A Londres, chez Thomas Woodward, au Croissant entre les Portes du Temple; et Jean Osborn, à la Boule d'Or, dans Pater Noster Row, près S. Paul. MDCCXLI, «Préface», non paginata.

(20) Ibid. 
Et comme on aime à connoître le Caractére de ceux dont il est fait mention dans un Livre qu'on lit, l'Auteur a bien voulu nous communiquer les Portraits de quelques personnes dont il parle dans cette Histoire» ${ }^{21}$. Questa indicazione, che almeno indirettamente costituisce una ulteriore conferma del fatto che la traduzione fu effettivamente eseguita in Inghilterra, è anche interessante per la collaborazione che fin dall'inizio si instaurò fra i traduttori e l'autore il quale, se non seguì direttamente il lavoro a causa della sua notoria ignoranza del francese, lo accompagnò tuttavia con le sue attenzioni. Scendendo nello specifico, le «Corrections» che Richardson fornì ai suoi traduttori sono quasi sicuramente alcune delle numerose correzioni che, a fronte delle critiche che gli erano state mosse e dell'inaspettato successo che l'opera aveva incontrato nelle settimane precedenti, lo scrittore inglese stava predisponendo in vista della terza edizione, che sarebbe uscita a metà marzo ${ }^{22}$; mentre le «Additions» sono costituite, essenzialmente, dai «Caractères», ovverossia dai ritratti delle quattro signore che nella prima parte del romanzo vanno a trovare Pamela, con l'intento di verificare di persona (e possibilmente di smontare) le belle qualità da più parti attribuite alla giovane allieva di Milady B***23. Questi «portraits» che non si troveranno nel testo inglese di Pamela se non a partire dalla sesta edizione, erano stati evidentemente già predisposti da Richardson che, prima di inserirli nel testo inglese, volle forse sperimentarli su un altro tipo di lettori, o che, più semplicemente, intese così gratificare i traduttori, il cui lavoro avrebbe certamente contribuito ad allargare il successo dell'opera, con queste primizie che, a loro volta, non avrebbero mancato di rendere la traduzione più interessante e, perciò, più degna di attenzione.

A parte questa eccezione, i due testi, perfettamente identici sia nella grafia che nell'impaginazione, riprendevano il testo della prima edizione inglese, anche se con qualche correzione rispetto ad essa, accogliendo quelle che, come abbiamo visto, Richardson propose loro, o facendo proprie alcune di quelle che, sulla scorta delle critiche ricevute, lo scrittore inglese aveva già inserito nella seconda edizione del suo romanzo; dalla quale però i traduttori non trassero i vari paratesti che Richardson, come è noto, vi aveva aggiunti. Nella traduzione francese del 1741 troviamo infatti solo i tre che erano contenuti nella prima edizione inglese: La «Preface», che qui diventa «Préface de l'Editeur», per distinguerla da quella, nuova, dei Traduttori (la stessa indicazione troviamo anche nell'edizione conservata alla British Library che, come abbiamo detto, non comporta la «Préface»); la lettera «A l'Editeur di Livre intitulé Pamela, ou la Vertu Recompensée», firmata J.B.D.F e la lettera «A mon digne Ami, Editeur de Pamela» che, come nell'edizione inglese, non appare firmata, benché provenga, con ogni probabilità, da William Webster, un amico e cliente di Samuel Richardson.

Essendo stata concepita e realizzata in Inghilterra, in linea di massima per un pubblico inglese, questa traduzione non poteva logicamente avere sul Continente, ed in modo particolare in Francia, un riscontro immediato: la prima allusione alla traduzione di Pamela sul Continente risale infatti, se non andiamo errati, all'inizio del 1742. Il 12 gennaio di quell'anno, ci fa sapere Henry Harrisse sulla scorta di una nota trovata in un documento conservato alla Biblioteca Nazionale di Parigi, «les syndics saisirent cinquante exemplaires de ce roman dans sa traduction française chez Guérin» ${ }^{24}$. Perché le cinquanta copie della traduzione francese di Pamela siano

(21) Ibid.

(22) Sulle correzioni via via apportate da Richardson al testo primitivo, cfr. T.C. D. EAvEs e B. D. KIMPEL, Richardson's Revisions of "Pamela", «Studies in Philology». 20, 1967, pp. 61-88.
(23) Cfr. Pamela, ou la Vertu Recompensée, ed. cit., lettre XXIII.

(24) H. Harrisse, L'Abbé Prévost. Histoire de sa vie et de ses ouvrages d'après des documents nouveaux, Paris, Calmann Lévy, 1896, p. 336. 
arrivate nella bottega dei Guérin, tra i più noti librai parigini dell'epoca, è cosa facile da intuire; perché queste copie siano state sequestrate è cosa assai più problematica. La traduzione sequestrata nella bottega dei Guérin era sicuramente quella uscita a Londra alla fine di ottobre dell'anno precedente. Il sequestro, operato dai «syndics», quindi dai responsabili dell'Arte, non dalla Polizia, è da attribuire al fatto che le copie erano state introdotte illegalmente, vale a dire senza pagare i droits d'entrée previsti dai Regolamenti francesi? Oppure l'intervento dei «syndics» era inteso a salvaguardare gli interessi di qualcuno che è impossibile identificare con precisione, ma che è ragionevole avvicinare a quegli stampatori che, forse con l'accordo di John Osborn, si accingevano a stampare, servendosi della sua adresse, la traduzione di Pamela destinata al mercato francese? Forse, all'epoca l'accordo non era stato ancora raggiunto, o forse i Guérin vollero approfittarne per offrire ai loro lettori una sorta di avant-goût, atto a suscitare la loro curiosità, con un'azione che però disturbò troppo l'operazione in corso e i delicati equilibri che regolavano i rapporti tra i librai parigini. Fatto sta che dopo quella data nessuno parlò più di Pamela, e della sua traduzione francese, fin oltre la metà dell'anno, con la sola eccezione della Bibliothèque raisonnée che in un articolo contenuto nel vol. 28, relativo ai mesi di aprile, maggio e giugno del 1742, presentò la traduzione di Pamela pubblicata a Londra da Thomas Woodward e John Osborn $^{25}$. La ragione sta nel fatto che fu solo verso la metà di giugno che le prime copie della traduzione francese di Pamela furono davvero messe a disposizione dei lettori parigini. La prima testimonianza in questo senso è contenuta in una lettera di Mme de Graffigny ad Antoine Devaux, datata «20 juin [1742]»:

Je viens de lire un roman anglois traduit par un Anglois. Il contient plus de neuf cent pages de très menue impression. Je l'ai lu en deux jours. C'est un monstre indéfinissable [...] Je ne saurois encore comprendre pourquoi je ne l'ai pas jeté par la fenêtre, et j'y ai passé une nuit entière ${ }^{26}$.

La testimonianza successiva è quella di un'altra lettrice, Mlle Delaunay (futura Mme de Staal), che in una lettera a certo M. d'Héricourt dell'indomani scriveva:

Quoique je ne sois guère en train de parler bagatelles, je vous demanderai si vous avez vu Pamela; c'est un roman Anglois traduit en François par un Anglois, mal écrit, chargé de détails qui devroient être fortement ennuyeux, présentant souvent des objets infiniment désagréables. Cependant j'ai lu les quatre tomes avec un attachement qui ne m'a pas permis de quitter, que je ne fusse au bout que j'ai trouvé avec regret ${ }^{27}$.

Dopo di allora le testimonianze sulla presenza a Parigi della traduzione francese di Pamela si susseguono, sempre più numerose, segno che la notizia della sua comparsa suscitò la curiosità di un numero crescente di lettori. Per tutte, valga quella dell'abbé Desfontaines, un noto giornalista dell'epoca, non particolarmente attento al genere romanzesco, che in una pagina delle sue Observations sur les Ecrits modernes, licenziata «ce 23 juin 1742», dopo aver avvertito che Pamela ou la vertu récompen-

(25) Cfr. Pamela, ou la Vertu récompensée; traduit de l'anglois. In- $8^{\circ}$, pages 819 , sans compter deux Préfaces \& deux Lettres. A Londres, chez Thomas Woodward \& John Osborn, 1741, in Bibliothèque raisonnée des Ouvrages des Savans de l'Europe pour les mois d'Avril, Mai \& Juin. Tome XXVIII, seconde Partie. Amsterdam, chez J. Wetstein, MDCCXLII, pp. 417-427.

(26) Cfr. Correspondance de Madame de Graf- figny, t. III (Ier octobre 1740- 27 novembre 1742 Lettres 309-490). Préparé par N. R. Johnson, Oxford, Voltaire Foundation, 1992, p. 318.

(27) Cfr. Lettres de Mlle De Launay (Mme de Staal) au chevalier de Menil, au Marquis de Silly et à M. d'Héricourt:; auxquells on a joint celles de M. de Chaulieu à Mlle de Launay. A Paris, chez Léopold Collin Libraire, 1806, t. II, p. 219. Cfr. anche H. HaRrisse, L'abbé Prévost cit., p. 337. 
sée, «Roman anglois [...] nouvellement traduit en françois [...] se vend[ait] chez les Guérins, rue S. Jacques, Didot \& Prault, Quai des Augustins, 4 vol. 6 livr. broc.», confessava che, nonostante la sua notoria «aversion pour les romans», incuriosito dal «prodigieux cours» che l'opera aveva conosciuto in Inghilterra, aveva anche lui impiegato «un jour entier à en lire les trois premiers volumes» e che avrebbe «dévoré» anche il quarto se improrogabili impegni di lavoro non ne l'avessero distolto ${ }^{28}$.

L'edizione messa in vendita verso la metà di giugno del 1742 dai Librai parigini ricordati dall'abbé Desfontaines non era però quella apparsa a Londra quasi otto mesi prima. Mentre la traduzione francese del 1741 comportava due tomi, rispettivamente di XX+351 e 458 pagine, quella posta in vendita a Parigi a metà del 1742 era suddivisa in quattro parti, talvolta rilegate in due tomi, rispettivamente di XXIII+273, 324, 298 e 302 pagine. Anche se la page de titre dava l'opera per stampata a Londra dallo stesso editore che aveva pubblicato la traduzione del 1741 (Pamela, ou la Vertu récompensée. Traduit de l'Anglois. Première [Quatrième Partie]. A Londres, Chez Jean Osborne, à la Boule d'Or, dans Pater-Noster-Row, près S. Paul, M.DCC. XLII), l'indicazione era manifestamente falsa: non solo perché il nome del supposto editore londinese era stato francesizzato, ma soprattutto perché l'esame della carta utilizzata conduce in modo inequivocabile (quand'anche ce ne fosse stato bisogno) al mondo degli stampatori francesi, anche se, per il momento, non è possibile essere più precisi ${ }^{29}$. Della traduzione francese di Pamela uscita nel 1742 con l'adresse «A Londres, chez Jean Osborne» sono giunte sino a noi parecchie copie, conservate in varie Biblioteche, in Francia e all'estero. Le filigrane della carta usata per le due copie conservate alla Biblioteca Nazionale di Francia rinviano entrambe a dei produttori di carta francesi e quindi, ragionevolmente, a degli stampatori dello stesso paese. Alle stesse conclusioni conduce l'esame della filigrana della copia conservata alla Bibliothèque de l'Arsenal. Riconducibili tutte e tre a stampatori francesi, queste copie non escono però tutte dalla stessa bottega: basta una lettura appena attenta per rendersene conto. Se la copia conservata all'Arsenal con la segnatura: $8^{\circ}$ BL 29924 (1-4) sembra essere identica a quella conservata alla Nazionale con la segnatura: Y2 11496-11499, la copia segnata Y2 11500-11503, pur presentando lo stesso numero di pagine per volume e gli stessi ornamenti di Y2 11496-11499, non è però ad essa identica, in quanto la stampa presenta, qua e là, delle piccole diversità rispetto a quella dell'edizione di riferimento (ad esempio, p. 2: «ce furent-là» per «ce furent là», p. 3: «ajouta t-il» per «ajouta-t-il»; p. 4 « sans cela?» per «sans cela.», «boëte» per «boête», «je sais» per «je sçais», ecc.). Françoise Weil dà conto di un'altra edizione datata 1742 che, pur presentando la stessa adresse e la stessa ripartizione in quattro parti, non comporta però lo stesso numero di pagine per parte (272, 320, 296, 295 rispettivamente) e che la studiosa qualifica di «lyonnaise» ${ }^{30}$. Si tratta evidentemente di una contrefaçon, stampata magari con l'accordo dello stampatore parigino, come talvolta succedeva.

Alan Dugald McKillop, allo scopo di suffragare l'origine inglese della prima versione francese di Pamela, ha fatto osservare che «the book is full of misprints» ${ }^{31}$. Un esame appena attento ed un confronto con l'edizione francese della traduzione fa in realtà vedere che il testo del 1741 non si caratterizza affatto per la sua scorrettez-

(28) P.-F. Guyot, abbé Desfontaines, Observation sur les écrits modernes, A Paris, chez Chaubert, à l'entrée du Quay des Augustins, M.DCC.XLII, t. XXIX, Lettre CCCXXIII, p. 70. Alla Pamela francese, ad ogni modo, l'abbé Desfontaines avrebbe dedicato una lunghissima, ed entusiastica Lettera il mese successivo.
(29) L'esame è stato eseguito alcuni anni orsono da Françoise Weil (cfr. F. WeIL, L'interdiction du roman et la Librairie 1728-1750, Paris, Aux Amateurs de Livres, 1986, p. 513).

(30) F. WeIL, op. cit., p. 482. Non è forse un caso che sia conservata a Mannheim.

(31) A. D. McKillop, op. cit., p. 93. 
za. Certo, esso presenta tratti che derivano chiaramente dalle abitudini tipografiche inglesi: in primis, l'abuso di maiuscole (non solo «Maître» e «Maîtresse», «Père» e «Mère», ma anche «Domestiques», «Fille-de-Chambre», «Chagrin», «Condition», «Bouse», «Gages», «Guinées», per citare solo i primi esempi offerti dalla lettura, comportano l'iniziale maiuscola); altre volte si nota qualche incertezza nell'uso degli accenti (l'accento circonflesso è spesso assente: «eut été» per «eût été», «pret» per «prêt», talvolta è usato dove il Francese non lo usa più: «plûtôt» per «plutôt»; l'accento acuto è usato spesso al posto di quello grave: «Pére», «Mére», «colére»; quest'ultimo è assente non di rado in parole che normalmente lo esigono: «Pere», «Mere», «fidele»); parole che terminano con una «ì, come «j'ai», «je finirai», «vrai», «quoi» nel testo inglese sono stampate una «y» finale; la desinenza «-és» dei participi passati plurali o di parole come «fossés» scritta «-ez» e si potrebbe continuare. Tuttavia queste incertezze grafiche non connotano solo l'edizione inglese, men che meno la rendono tale da considerarla, come riteneva McKillop, trascurata e piena di refusi. Chi ha una certa dimestichezza con la stampa settecentesca, sa che all'epoca la grafia era ancora piuttosto incerta, che tra un testo e l'altro si trovano differenze a volte notevoli, e che differenze ed incertezze si trovano anche all'interno dello stesso volume o tra i vari volumi della stessa opera, proprio come succede per i due tomi della traduzione francese di Pamela apparsi in Inghilterra: il secondo (quello che porta l'adresse del solo Jean Osborn) presenta una grafia decisamente più moderna e curata di quello stampato per conto di Thomas Woodward da William Bowyer; per cui si può tranquillamente concludere che il testo inglese della traduzione di Pamela non deve affatto ritenersi particolarmente corrotto.

I responsabili delle varie edizioni francesi, al di là di una revisione grafica e tipografica che lo adeguasse alle abitudini francesi, hanno del resto seguito passo passo il testo proposto dall'edizione inglese, recependo a volte le sue lezioni anche quando non erano né corrette né moderne (ad esempio conservando «souvien-toi» per «souviens-toi», «que tu prenne» per «que tu prennes», «bienfaite» per «bien faite», ecc.), altre volte sostituendo ad una lezione incerta una lezione altrettanto incerta quando non addirittura scorretta. Il responsabile della copia conservata alla Biblioteca Nazionale di Parigi sotto la segnatura Y2 11496-11499 ha per esempio corretto «Elle nous a laissez tous dans une extrème affliction» con «Elle nous a laissé tous dans une extrême affliction» ricorrendo ad una costruzione (quella del participio passato non accordato davanti ad un attributo) che nel Settecento era sentita ormai altrettanto se non più arcaica di quella usata dallo stampatore inglese. Altrettanto discutibile è la correzione che egli ha apportato ad un'altra frase: «Montre-moi quels progrès tu as faits» chiede ad un certo punto $\mathrm{M}$. B... a Pamela; l'editore francese ha corretto in «Montre-moi quels progrès tu as fait». Decisamente fuori luogo pare invece il modo in cui il responsabile dell'edizione parigina ha corretto la frase seguente: «Tout le monde dit que tu es devenue grande et bienfaite; quelques uns ajoutent que tu es fort jolie; \& en vérité, si tu n'étois pas ma fille, je l'aurois crû aussi lors que je te vis la derniére fois» con «je l'aurois cru aussi-tôt que je te vis la dernière fois il y a six mois» con un intervento non solo non necessario ma addirittura inopportuno in quanto cambia, ed in peggio, il senso, del resto chiarissimo, della frase originale. Gli interventi eseguiti sul testo dell'edizione inglese sono, peraltro, rari: a parte la correzione di qualche refuso (tipo «tu ne te laisses pas entraîner» per «tu ne le laisses pas entraîner», «Pourquoi te donneroit-il ...?» per «Pourquoy te donneroit ...?»), l'unico intervento degno di nota che si incontra nelle prime pagine del primo volume è il seguente: mentre nella versione londinese, Pamela nella quarta lettera scriveva con soddisfazione ai propri genitori che Mylady Davers nel mese trascorso in casa di Mr. B... «a pris beaucoup de connoissance d'[elle]» e le ha «conseillé d'être toujours très reservée», in quella parigina Pamela scrive che «Mylédy Davers [...] a eu beaucoup 
d'égards pour [elle]», con una correzione che forse rende la frase più elegante ma che esprime certamente meno bene il senso della frase originale («has taken great notice of me») e, più in generale, di tutto il periodo.

Nell'opera di diffusione della Pamela di Richardson tramite la sua traduzione francese, agli editori parigini si affiancarono però, in quello stesso anno, anche gli editori olandesi che all'epoca costituivano, come è noto, l'altro, e forse più importante polo editoriale e librario dell'intera Europa. Ad una data che è difficile stabilire con precisione ma che, a quanto si può capire, si situa contemporaneamente alla comparsa nelle librerie francesi della traduzione di Pamela messa a punto dai traduttori inglesi e stampata, forse, da uno o più editori francesi, i Libraires associés di Amsterdam misero sul mercato - un mercato che naturalmente non si limitava solo a quello olandese - una loro edizione della traduzione francese del capolavoro richardsoniano. La ragione, almeno apparente, di questa operazione stava nel fatto che l'edizione inglese era piena di errori e presentava una traduzione che rendeva male il senso del testo originale. Alla fine dell'articolo con il quale la Bibliothèque britannique segnalava la traduzione francese, fatta da un certo «Mr. De M*** [per lo più identificato con Eléazar Mauvillon], e apparsa ad Amsterdam nel 1743, di Anti-Pamela, or Feign'd Innocence detected in a series of Syrena's Adventures, una delle più fortunate parodie del romanzo di Richardson ${ }^{32}$, l'autore faceva infatti notare che: «les deux traductions de ces Romans de Pamela, \& d'Anti-Pamela, ne sont nullement bien écrites, \& se sentent fort souvent du Langage altéré des Lieux qu'habitent leurs Auteurs», precisando che «la premiere [vale a dire quella di Pamela] est fort irréguliere dans l'édition de Londres, \& que par cette Raison l'on a été obligé de rajuster tant bien que mal en beaucoup d'endroits des Editions d'Amsterdam» ${ }^{33}$. Di fatto, gli editori olandesi intesero sfruttare il sempre più grande successo che l'opera di Richardson aveva riscosso, ed ancora stava riscuotendo al momento in cui procedettero alla ristampa della traduzione inglese; ad una data che, come dicevamo, non è dato determinare con precisione ma che aiuta a situare con sufficiente approssimazione quanto è detto nell'«Avis des nouveaux Editeurs» che apre il primo volume:

La Bibliotheque Britannique $e^{34}$ nous apprend que Mr. Richardson, Auteur de Pamela, ne s'étoit proposé d'en donner que ces deux volumes ${ }^{35}$; mais qu'il la continuera sur des Mémoires qu'il a, \& qu'il n'auroit peut-être jamais publiés si quelques Libraires de Londres, amorcés par le prodigieux succés de ces deux Volumes, dont il s'est fait cinq Editions dans un an, ne l'y avoient forcé, en promettant au Public une suite sous le titre de Pamela dans la Grandeur, qui ne peut être que le fruit de l'imagination de celui qui y travaille, au lieu que la sienne contiendra des faits réels ${ }^{36}$.

Sappiamo che «the first public intimation that Richardson gave of his intention to continuate Pamela beyond the first two volumes was in the form of an advertisement of the fourth edition of Pamela Vols. I and II.» ${ }^{37}$, vale a dire a metà del 1741, visto che la quarta edizione dell'opera uscì il 5 maggio 1741, e che i tomi III e IV di Pamela, or Virtue Rewarded uscirono, seppure con la data del 1742, ai primi di dicem-

(32) L'opera, comparsa a Londra già alla fine del 1741, è tradizionalmente attribuita ad Eliza Haywood (cfr. W. M. SALE, Samuel Richardson cit., p. 116)

(33) Cfr. Bibliothèque britannique cit. Tome vingt-deuxieme. Seconde Partie. A La Haye, chez Pierre De Hondt, M.DCC.XLIV, p. 365.

(34) Tom. XVII. Part. II. Pag. 222 e\&.(N. d. A.)

(35) «Ce qu'on peut conclure aussi de la fin du
II. Tome» (N.d.A.), che porta in effetti la parola «Fin».

(36) Cfr. Pamela, ou la Vertu recompensée. Traduit de l'Anglois. Tome premier. A Amsterdam, Aux Dépens de la Compagnie, M DCC XLII, «Avis des nouveaux Editeurs», n. n.

(37) W. M. SALE, op. cit., p. 27. 
bre di quello stesso anno, dopo che il 22 settembre era uscita la quinta edizione dei tomi I e II, alla quale l'«Avis des nouveaux Editeurs» fa pure riferimento. L'«Avis» fu di conseguenza scritto tra la fine di settembre ed i primi di dicembre, ad ogni modo prima della fine del 1741.

In linea di massima, l'edizione olandese della traduzione francese di Pamela sarebbe dovuta essere stata messa in circolazione nei primi mesi del 1742. Di essa tuttavia non è, a quanto sembra, apparsa alcuna notizia sulla stampa francese, abbastanza attenta invece all'edizione parigina, per tutto il 1742 ed anche oltre. Abbiamo infatti incontrato il primo riferimento esplicito all'edizione olandese del 1742 nella «Lettre à Madame **** sur le Livre intitulé Pamela ou la Vertu récompensée, traduit de l'Anglois. Edit. d'Amsterdam. MDCCXLII» che una nota dice «écrite à Lausanne en 1742 dans le tems que l'Ouvrage paroissoit ou du moins peu de tems après $\rangle^{38}$ anche se il Journal helvétique, nel quale l'abbiamo trovata, l'ha pubblicata solo nell'agosto del 1743. L'edizione olandese della traduzione francese di Pamela non uscì comunque prima della metà del 1742 .

Dipese ciò dal fatto che la revisione di una traduzione che, come abbiamo visto, la Bibliothèque britannique aveva considerato «fort irréguliere» richiedette molto più tempo di quanto l'autore dell'«Avis» avesse ipotizzato in un primo momento? Un confronto con l'edizione inglese, dalla quale gli editori olandesi sono sicuramente partiti (anche perché all'epoca l'edizione francese non era ragionevolmente ancora apparsa) parrebbe non convalidare questa ipotesi. Le correzioni che gli editori olandesi hanno apportato alla traduzione predisposta in Inghilterra, molto più numerose di quelle apportate allo stesso testo dagli editori parigini, non sono tuttavia, né per quantità né per qualità, tali da giustificare un simile ritardo. Gli editori olandesi hanno prima di tutto provveduto ad adeguare la grafia dell'edizione londinese alla prassi continentale (per esempio usando meglio i vari accenti, riunendo parole come «lorsque», o «quoique» che nell'edizione inglese erano divise, riducendo sistematicamente ad «i» le «y» finali di parola) oppure correggendo alcune grafie («souvent» per «souvant», «cependant» per «cepandant», «encore» per «encor»); hanno ridotto le numerose maiuscole del testo inglese (meno però di quanto avessero fatto gli editori parigini, in quanto un certo numero di maiuscole sono conservate: per «Maitre» e «Maîtresse», per «Père» e «Mère», ma anche per «Dame» e «Demoiselle», «Enfant» e «Créature» o per «Lettre»); infine hanno provveduto a correggere, con maggiore attenzione - occorre dirlo - degli editori parigini, i non frequentissimi refusi dell'edizione londinese (ad esempio «que tu prennes» per «que tu prenne», «tu ne te laisses pas entraîner» per «tu ne le laisses pas entraîner», «tu nous aimes» per «tu nous aimez», «que tu nous fais» per «que tu nous fait», ecc.).

A differenza degli editori parigini che, come abbiamo visto, hanno seguito, tranne qualche eccezione, passo passo il testo londinese, gli Editori olandesi sono intervenuti anche sul testo dell'edizione inglese per renderlo, oltre che più corretto e vicino alle abitudini continentali, anche più scorrevole e stilisticamente migliore. È l'obiettivo dichiarato di alcune semplificazioni apportate al testo di partenza. Nella traduzione londinese, rendendo conto dell' atteggiamento che Mr. $\mathrm{B}^{* * *}$ assume nei suoi riguardi subito dopo la morte della madre, Pamela scrive che rivolgendosi a lei, Mr. B*** le ha detto: «Pour toi, Pamela, ajouta-t-il, en me prenant la main (oui en verité, il me prit la main en presence de toutes les autres filles) je veux etre ton ami pour l'amour de ma chere Mére», mentre il testo olandese presenta soltanto «Pour toi, Pamela, ajouta-t-il en me prenant la main en présence de toutes les autres filles, 
je veux être ton ami pour l'amour de ma chère Mère» con una semplificazione che rende la frase indubbiamente più scorrevole e, se si vuole, anche più consona alla Pamela delle prime lettere, ma che si allontana dal testo di partenza che comportava la sottolineatura presente nell'edizione inglese ${ }^{39}$. Un po' più oltre, Pamela scrive che Mr. B*** «a fait présent à chacun [des domestiques] d'un an de Gages» mentre «Par rapport à [elle], comme [elle] n'avoi[t] point encor de Gages [...] il a ordonné à la Menagère de [la] mettre en doeuil comme les autres \& il [lui] a donné de sa propre main quatre Guinées d'Or, \& quelques pieces d'Argent». Gli editori olandesi da un lato hanno evitato la ripetizione di «gages» sostituendo la frase «Par rapport à moi, comme je n'avois point encor de Gages» con «Par rapport à moi, comme je n'en avois point encore eu», rendendo la frase indubbiamente più scorrevole; dall'altro hanno sostituito «Guinées d'Or» con il semplice «Guinées», forse ritenendo il complemento di specificazione un'inutile ridondanza, senza rendersi però conto che «d'or» faceva da utile pendant, almeno dal punto di vista stilistico, all'espressione «d'argent» che segue subito dopo ${ }^{40}$. Altre volte l'intervento degli Editori olandesi intende, anche se non sempre con soluzioni del tutto convincenti, adeguare meglio il testo di partenza alla sintassi francese: così se è logico che correggano la frase: «Paroles fatales, qu'il t'ait dit: fais ce que tu dois \& que tu n'en es que plus à mon gré» con «Paroles fatales, qu'il t'ait dit: fais ce que tu dois \& que tu n'en seras que plus à mon gré» in quanto la consecutio è meglio rispettata nella seconda frase che nella prima, meno convincente è la correzione della frase: «J'étois au chevet de son lit, pleurant \& sanglotant» con «J'étois au chevet de son lit, pleurante \& sanglotante» con la quale Pamela descrive il suo atteggiamento di fronte alla sua amata padrona che sta per andarsene. Sono comunque, come si vede, tutte correzioni di poco conto, che non hanno dovuto richiedere molto tempo a colui al quale la correzione del testo inglese è stata affidata; tanto più che queste correzioni, non numerose neppure nelle pagine iniziali dell'ope$\mathrm{ra}$, si fanno ancora meno frequenti a mano a mano che si procede con la lettura ed il confronto. Per cui devono essere state altre le ragioni che ritardarono l'uscita dell'edizione olandese fino alla metà del 1742, anche se non sappiamo bene quali.

Stando al numero degli esemplari pervenuti sino a noi, è stata tuttavia proprio questa edizione, con le sue varie riedizioni e contraffazioni, che contribuì sopratutto alla diffusione dell'opera di Richardson in Europa. Il 1743 vide non solo una ristampa dell'edizione del $1742^{41}$ ma anche una sua probabile contrefaçon belga che, pur riprendendo il testo olandese, si presentava tuttavia in maniera assai diversa: dopo l'«Avis des nouveaux Editeurs», la «Préface du Traducteur», la «Préface de l'Editeur», la lettera «A l'Auteur du Livre intitulé Pamela, ou la Vertu Recompensée» e

(39) «For you, Pamela, (and took me by the hand; yes, he took my hand before them all) for my mother's sake, I will be a friend for you».

(40) Anche in questo caso, traducendo con una certa libertà il testo inglese: «he has given mourning and a year of wages to all my lady's servants; and I having no wages as yet, my lady having said she should do for my as I deserved, ordered the housekeeper to give me mourning with the rest; and gave me with his own hand four golden guineas, and some Silver».

(41) Pamela, ou la Vertu recompensée. Traduit de l'Anglois. Tome premier [Quatriéme]. A Amsterdam, Aux dépens de la Compagnie. MDCCXLIII. Quattro tomi rispettivamente di pp. 216 (+ XXIII di testi introduttivi), 264, 248 e 247 (BN:Y2 11508 11511). H. Harrisse (op. cit., p. 341) dà però anche conto di una Pamela, ou la Vertu récompensée, traduit de l'anglois. Nouvelle édition, enrichie de Figures. A Amsterdam, aux dépens de la Compagnie. MDCCXLIII, che non ha visto personalmente ma che, sulla base di quanto detto da Cohen nel suo Guide de l'amateur de livres à figures du XVIII siècle: «In-douze, quatre volumes, quatre frontispices (le même pour chaque volume) et vingt-neuf figures par Pont», presenta in modo da far pensare alla Troisième édition, quella del 1744. Da un lato, questo giustificherebbe il fatto che le planches di Punt inserite in quest'ultima edizione portano la data del 1742, dall'altro confermerebbe l'indicazione data dagli Editeurs de Hollande nell'«Avis» del 1744 secondo cui a quella data erano uscite tre traduzioni olandesi di Pamela. 
quella «A Mon digne Ami l'Editeur de Pamela», per complessive 24 pagine non numerate, la Première Partie del Tome I dell'opera finiva a pag. 294 mentre la Seconde Partie comprendeva 336 pagine. A sua volta la Troisième Partie (prima del Tome II) comportava 328 pagine, mentre la Quatrième ne comportava 320. Si tratta di una edizione tipograficamente assai ben curata: ogni tomo comporta un bel frontespizio, dei titoli in rosso e nero ed una elegante vignette rappresentante due pescatori su una barca che tirano su la rete ${ }^{42}$. L'anno successivo i Libraires associés di Amsterdam pubblicarono una bellissima «Troisième Edition, revue, \& enrichie de Figures en Tailles douces» in due volumi rispettivamente di 480 e 491 pagine. Oltre ad offrire un testo tipograficamente ancora più corretto (che riprendeva tuttavia quello del 1742) ed una veste tipografica ancora più accurata, essa era impreziosita da quattordici elegantissime planches (sette per il tomo I e sette per il tomo II) oltre ad una quindicesima che precede la page de titre sia del primo volume che del secondo ${ }^{43}$. L'esemplare conservato alla Bibliothèque Publique et Universitaire di Ginevra, che abbiamo potuto consultare grazie alla cortesia del suo ancien conservateur Jean-Daniel Candaux, comporta solo due volumi, quelli che corrispondono ai primi due tomi dell'opera di Richardson, i soli ad aver circolato fino ad allora sul Continente in traduzione francese. È tuttavia assai probabile che la nuova edizione comportasse anche la traduzione dei tomi III e IV di Pamela, usciti, se l'ipotesi formulata dianzi è corretta, l'anno precedente in quella che deve chiamarsi la seconda edizione olandese. Non solo alla fine del secondo volume c'è infatti l'indicazione «Fin du Tome II.» al posto del semplice «Fin», come nelle precedenti edizioni da noi consultate, ma in quello stesso anno a Liège, presso J.-F. Bassompierre e J. Delorme de la Tour, apparve quella che fu senza dubbio una contrefaçon dell'edizione olandese, anch'essa in due tomi, indicati nelle pages de titre come III e IV, ripartiti, come i tomi I e II in due Parties ciascuno, e comportanti rispettivamente pagine 408 per la Cinquième Partie, 410 per la Sixième, 424 per la Septième e 399 per la Huitième ${ }^{44}$. Vero è tuttavia che l'edizione olandese del 1744 era aperta da un «Avis des Editeurs de Hollande», che non figurava, per quanto ne sappiamo, in alcuna edizione precedente: dopo aver ricordato il grande successo che l'opera di Richardson aveva conosciuto sia in Inghilterra dove «il s'est fait [...] six Editions dans un peu plus d'un an», sia sul Continente dove «dans un temps guères plus long on en a fait quatre Editions [...] une en Angleterre, \& trois en Hollande, en comptant celle-ci» ${ }^{45}$, e aver fatto osservare che in effetti «la belle Pamela» poteva «servir de modèle à la jeunesse de son sexe par les diverses qualités morales dont elle étoit ornée», gli «Editeurs de Hollande» citavano due brani contenuti entrambi nel secondo tomo, senza alcun riferimento al seguito della storia di Pamela ${ }^{46}$.

(42) Pamela, ou la Vertu Récompensée. Traduit de l'Anglois. Tome I. [II.] Première [Quatrième] Partie. A Londres, \& se vend à Liège, chez J.-F. Bassompierre, Imprimeur \& Libraire au Neuvice, $\&$ J. Delorme de la Tour, Marchand Libraire, près du Palais. MDCCXLIII (BN: Y2 11404-11607 e Arsenal: $8^{\circ}$ BL 29925 1-4). Sebbene la (falsa) indicazione di «Londres» potesse far pensare ad un ricorso al testo apparso a Londra nel 1741, il testo proposto da Bassompierre e Socio era in realtà quello dell'edizione olandese.

(43) Pamela, ou la Vertu récompensée. Traduit de l'Anglois. Troisième Edition, revue, \& enrichie de Figures en Tailles-douces. Tome I. [II.]. A Amsterdam, Aux dépens de la Compagnie. MDCCXLIV
(BPU di Ginevra: Su 1382)

(44) Pamela, ou la Vertu Récompensée. Traduit de l'Anglois. Tome III. [IV.] A Londres, \& se vend a Liège, Chez J.-F. Bassompierre Imprimeur \& Libraire en Neuvice \& J. Delorme de la Tour, Marchand Libraire, près du Palais, MDCCXLIV (Arsenal: $8^{\circ}$ BL29925 5-8).

(45) Si sarà notato che, per motivi peraltro comprensibili, gli «Editeurs de Hollande» non fanno alcuna menzione delle edizioni (o contrefaçons?) francesi che erano succedute a quella inglese.

(46) Pamela, ou la Vertu récompensée [...]. Troisième Edition cit., t. I. «Avis des Editeurs de Hollande», pp. 2-3. 
Su questa traduzione non abbiamo ad ogni modo, per il momento, nulla di certo: né per quanto riguarda le circostanze nelle quali essa è nata ed è stata eseguita, né per quanto riguarda l'autore o gli autori. Nulla al riguardo dice il breve «Avertissement sur ces nouveaux volumes» che abbiamo trovato in apertura al terzo volume della contrefaçon belga, apparso con la data del 1744, in quanto esso, più che spiegare le ragioni o le circostanze della traduzione, mira ad invogliare il lettore, facendo intravedere una Pamela ancora più interessante di quella che il lettore ha conosciuto «Fille», tramite una lettura perfettamente in linea con i propositi che avevano spinto Richardson a proseguirne la storia:

On peut assurer que cette suite de Pamela est beaucoup plus intéressante que le commencement. Pamela, ou Madame B***, spirituelle, éclairée, \& souvent en compagnie de Personnes qui s'entretiennent de matières des plus utiles, est toûjours consultée, \& toûjours elle répond d'une manière satisfaisante. Elle fait aussi des Portraits, \& peint en Maître. Telle encore qu'on l'a vuë Fille, on la trouve ici Femme. La grandeur, l'opulence, n'altèrent en rien son caractère: même humilité, même sagesse, même bienfaisance, même amour pour Dieu, même respect pour ses Père \& Mère; beau \& rare modèle enfin des sentimens qui devroient toûjours animer les Personnes que la Providence a appellées à l'état du Mariage! ${ }^{47}$

Seguiva una «Préface» che, di fatto, era la traduzione della «Preface» posta da Richardson in testa alla sua continuazione di Pamela, apparsa, come abbiamo detto, in due tomi a Londra alla fine del 1741. L'edizione belga, pur essendo tipograficamente ben curata, non comportava, diversamente da quella olandese, alcuna planche. Le pages de titre dei due tomi presentavano la stessa vignette che ornava le pages de titre dei primi due tomi: sicché, come nel caso dell'edizione olandese, i quattro tomi erano destinati a costituire un tutt'uno ed a consacrare anche sul Continente un'opera alla quale l'enorme successo incontrato sia al di là che al di qua della Manica aveva conferito la natura e il ruolo del vero capolavoro.

Con l'edizione olandese del 1744, e con la sua contrefaçon belga, può ritenersi conclusa la prima fase della diffusione, attraverso l'obbligato tramite della traduzione francese $^{48}$, di Pamela sul Continente, così come può ritenersi conclusa la storia dello straordinario successo che il capolavoro di Richardson conobbe anche al di qua della Manica. Al pari delle reazioni che l'opera suscitò sui lettori, e di cui è rimasta traccia nei giornali e nelle corrispondenze dell'epoca, anche le edizioni conobbero, sul Continente come in Inghilterra, un periodo di stasi: a seguito dapprima della messa all'Indice, da parte della Chiesa Cattolica, della traduzione francese di Pamela, poi dell'entusiasmo suscitato da Clarissa Harlove, i primi tomi della quale uscirono alla fine del 1747. L'attenzione per Pamela non venne tuttavia mai meno; ne fanno fede le numerose riedizioni che videro la luce nella seconda metà del Settecento: da quella apparsa con l'adresse (probabilmente falsa) di «A Amsterdam, Chez Scheuders» nel 1768 e che si vendeva «A Paris, Chez Laurent Prault, Quai des Augustins, \& Desaint, rue du Foin», e che riprendeva l'edizione parigina del $1742^{49}$, a quella apparsa a Rou-

(47) Ibid., t. III, p. 3.

(48) In effetti, anche se si fecero e si pubblicarono traduzioni in altre lingue (cfr. Pamela oder die belobnte Tugend eines armen aber wunderschönen Dienstmädchens in ibren eigenen, auf Wabrbeit und Natur gegründeten Briefen entdecket, ans Licht gestellet und nach der 4ten Auflage aus dem Englischen übersetzt [ad opera di Johann Mattheson?], 6 parti in 4 volumi, Amburgo, Francoforte e Lipsia, 1742; Pamela oder die belobnte Tugend. Aus der sechsten vermebrten Englischen Auflage in das Deutsche übersetzt und mit Kupfern gezieret, 4 vol. Lipsia 1743; Pamela, ovvero la Virtù premiata, 4 vol. Venezia 1744-1746; ecc.) fu soprattutto per il tramite della traduzione francese che Pamela venne soprattutto letta sul Continente.

(49) Pamela, ou la Vertu Récompensée. Traduit de l'Anglois. Première [Quatriéme] Partie. A Amsterdam, Chez Schreuders. Et se vend à Paris, Chez Laurent Prault, Quay des Augustins, à la Source des 
en presso la Veuve de Pierre Dumesnil nel 1782, che riprendeva invece l'edizione olandese, e che nel titolo indicava per la prima volta sia il nome dell'autore dell'opera che quello del suo [presunto] traduttore ${ }^{50}$; da quella apparsa l'anno seguente a Ginevra grazie a Nouffer de Rodon tra le Euvres de Richardson, di cui costituiva i primi otto volumi e che riprendeva l'edizione olandese del 1744, comprendente tanto la prima Pamela che la sua continuazione ${ }^{51}$, a quella apparsa nel 1784 all'interno delle Euvres choisies dell'abbé Prévost, delle quali costituiva i tomi XVII e XVIII, e che, pur con qualche intervento correttivo, riproponeva invece il testo della traduzione apparsa a Parigi nel $1742^{52}$; per finire con quella in dodici volumi «avec Figures» apparsa nel 1793 a Parigi «chez Lepetit» che, riprendendo l'edizione olandese del 1744, offriva per la prima volta al pubblico francese la traduzione completa dell'opera di Richardson ${ }^{53}$. Tenendo conto delle indicazioni che abbiamo incontrato nei cataloghi delle principali biblioteche epopee ed extraeuropee che abbiamo consultato, e di cui non abbiamo però ancora potuto verificare del tutto l'attendibilità, si dovrebbe superare la quindicina di edizioni: una testimonianza inoppugnabile del grande successo che la prima opera di Richardson, di solito un po' trascurata dai critici e dagli storici, conobbe in Francia e del forte impatto che essa ebbe sia sui lettori ${ }^{54}$, sia, come avremo modo di mostrare meglio altrove, sull'evoluzione stessa che la letteratura, quella legata al romanzo ma non solo, conobbe a seguito del suo impatto con la Pamela di Richardson.

Sciences, \& Desaint, rue du Foin, M. DCC. LXVIII (BN: Y2 11512-11515 e Y2 9708 [che comprende però solo la Troisiéme e la Quatriéme Partie]).

(50) Pamela, ou la Vertu Récompensée. Traduit de l'Anglois de Richardson par M. l'Abbé Prévost. Tome premier [Quatriéme], Rouen, chez la Veuve de Pierre Dumesnil, rue Poterne \& Labbey, proche du Collège, M.DCC. LXXXII (BN: Y2 11520-11521 e Arsenal: $8^{\circ}$ BL 29926 (1-4).

(51) Euvres de Richardson. Tome I. [VIII.] Pamela, ou la Vertu récompensée. Traduit de l'Anglois par M. l'abbé Prévost. Tome premier [huitième]. A Genève, chez Nouffer de Rodon \& Compagnie, Imprimeur-Libraire, 1783 («Bibliothèque ou choix des meilleurs romans anglois». Tome seizième [vingt-troisième]) (BN: Réserve Y2 12218-12225 e BPU di Ginevra: S 19929)

(52) Pamela, ou la Vertu récompensée; traduit de l'Anglois, par l'Abbé Prévost: avec Figures. Tome premier [deuxième]. A Amsterdam, Et se vend à Paris, rue et Hôtel Serpente, M. DCC. LXXXIV, in Euvres choisies de l'Abbé Prévost, avec Figures. Tome dix-deptième [ dix-huitième] (BN: Réserve: Y2 1815-1816).

(53) I cataloghi di Biblioteche francesi e straniere che abbiamo consultato citano anche altre edizioni della traduzione francese di Pamela, che non abbiamo ancora potuto vedere. Ad esempio, il NUC dà come presente nella Biblioteca dell'Università di Washington, Seattle, una copia di «Pamela, ou la vertu récompensée. Traduit de l'anglois de Richardson par M. l'Abbé Prévôt. Amsterdam, aux dépens de la Compagnie, 1765, 8 vol. in 4.» che, se le indicazioni fossero esatte, costituirebbe non solo un'altra riedizione della traduzione completa di Pamela, ma anche la prima esplicita attribuzione della traduzione all'abbé Prévost. Altre indicazioni al riguardo sono fornite dal sito Centre/cet/trad/ richardson dell'Università di Metz, dal quale sembra venire una conferma che la prima traduzione completa dell'opera di Richardson apparve ad Amsterdam «Aux dépens de la Compagnie» nel 1743 (la Biblioteca dell'Università di Yale, New Haven, ne possiede una copia in 8 volumi) e dà anche conto sia di una ripresa, da parte di Anne-Catherine Bassompierre, probabile figlia ed erede di quel J-.F. Bassompierre che abbiamo incontrato precedentemente, di una Pamela in «Quatre tomes en $8^{\circ} \gg$ che farebbe pensare ad una ristampa della sola prima parte di Pamela piuttosto che dell'intera opera, sia di una ripresa da parte della Veuve de Pierre Dumesnil e Labbey nel 1787 della traduzione completa di Pamela dagli stessi editori già pubblicata una prima volta nel 1782 (BL 1568/256).

(54) L'analisi svolta a suo tempo da Daniel Mornet sui cataloghi di cinquecento biblioteche private francesi del Settecento ha fatto vedere che, dopo la Nouvelle Héloïe di Rousseau, Pamela era l'opera che comportava il maggior numero di presenze e che essa era l'opera straniera più acquistata dai lettori francesi del Settecento, davanti al Tom Jones di Fielding e alla Clarissa dello stesso Richardson (D. MORNET, Les enseignements des bibliothèques privées (1750-1780), in «Revue d'histoire littéraire de la France», XVIII (1910), p. 461). 


\section{Il traduttore}

La prima traduzione francese di Pamela è stata, ed è ancora tradizionalmente attribuita all'abbé Prévost che di Richardson, negli anni successivi, tradusse in effetti, o per meglio dire adattò al gusto francese, gli altri due capolavori: Clarissa, or the History of a young Lady che, a partire dal 1751 iniziò ad apparire sotto il titolo Lettres anglaises ou Histoire de Miss Clarisse Harlove e The History of sir Charles Grandison che a partire dal 1755 iniziò invece ad apparire sotto il titolo Nouvelles Lettres anglaises, ou Histoire du chevalier Grandisson. L'attribuzione della traduzione francese di Pamela all'abbé Prévost è tuttavia, occorre precisarlo subito, abbastanza tardiva: alcune indicazioni rinviano al 1782 ( $\mathrm{fu}$ infatti in quell'anno che a Rouen, per i tipi della Veuve di Pierre Dumesnil, apparve Pamela, ou la Vertu récompensée. Traduit de l'Anglois de Richardson par M. l'abbé Prévost, una ennesima riedizione del romanzo inglese, oltretutto nella sua versione completa, il cui titolo conteneva - ed era a mia conoscenza la prima volta - un esplicito riferimento al traduttore; altre indicazioni, che però non siamo ancora riusciti a controllare, farebbero risalire la prima attribuzione della Pamela francese all'abbé Prevost al 1765 (fu infatti in quell'anno che sarebbe stata stampata «Aux dépens de la Compagnie des Libraires d'Amsterdam» Pamela, ou la Vertu récompensée. Traduit de l'Anglois de Samuel Richardson par l'abbé Prévôt, un esemplare della quale è conservato nella Biblioteca della Washington University di Seattle), cioè a poco più di vent'anni dopo la sua prima comparsa in libreria. $\mathrm{Fu}$ ad ogni modo a partire dal 1783 (anno in cui apparve l'edizione ginevrina della traduzione francese di Pamela, presentata anch'essa come «traduit[e] de l'Anglois de Richardson par M. l'Abbé Prévost», e soprattutto a partire dal 1784 quando la traduzione francese di Pamela fu inserita (assieme a quelle di Clarissa e di Grandison) nelle Euvres choisies dell'abbé Prévost, che il nome dell'autore di Manon Lescaut fu, quasi automaticamente, associato alla traduzione francese di Pamela. Nella prima metà dell'Ottocento, A.-E. Barbier nel suo celebre Dictionnaire des ouvrages anonymes e J.-M. Quérard nella sua altrettanto celebre France littéraire attribuirono a Prévost, assieme alle altre due, anche la traduzione di Pamela ${ }^{55}$. La stessa cosa fece, nella seconda parte del secolo, H. Harrisse nel suo documentatissimo studio sulla vita e le opere dell'abbé Prévost ${ }^{56}$. L'attribuzione della traduzione francese di Pamela a Prévost divenne presto un luogo comune: ne fanno fede, tra le altre, le celebri Histoires de la Littérature française pubblicate tra la fine dell'Ottocento e l'inizio del Novecento rispettivamente da Gustave Lanson e da Joseph Bédier et Paul Hazard ${ }^{57}$. Sulla stessa linea si collocano anche le Storie più recenti: da quella, ormai classica, di André Lagarde et Laurent Michard a quella di Pierre-Georges Castex e Paul Surer per finire con quella, più recente, di Michel et Jeanne Charpentier ${ }^{58}$, per limitarsi ad

(55) Cfr. A.-E. BARBIER, Dictionnaire des ouvrages anonymes. Troisième édition revue et corrigée par MM. Olivier Barbier, René et Paul Billard, Paris, Maisonneuve \& Larose, 1964, t. IIIe, col. 764; e J.-M. QUÉRARD, La France littéraire ou Dictionnaire bibliographique des savants, historiens et gens de lettres de la France, Paris, Maisonneuve \& Larose, 1964, t. VIIIe, p. 30.

(56) H. HARRISSe, L'abbé Prévost, cit., p. 336. «Prévost commence à traduire Richardson» (con esplicito riferimento a Pamela), p. 337: «la traduction de Prévost serait à certains égards plus complète que les éditons anglaises faites jusqu'alors», p. 417: «Liste complète de ses œuvres».
(57) Cfr. G. Lanson, Histoire de la littérature française. Remaniée et complétée pour la période 1850-1950 par Paul TufFrau, Paris, Hachette, 1961 (prima edizione 1894), p. 676: «c'est lui qui plus tard met en français Pamela (1742) et Clarisse Harlowe (1751)» e J. BÉDIER et P. HAZARD, Littérature française. Nouvelle édition refondue et augmentée sous la direction de P. MarTino, Paris, Librairie Larousse, 1949 (prima edizione 1923-24), t. II, p. 49: «errant de la Belgique à l'Allemagne, il donne quelques romans et la traduction de Pamela (1742)».

(58) Cfr. A. Lagarde \& L. Michard, Les grands auteurs français du programme: XVIII' Siècle, Paris, Bordas, 1963, p. 70; P.-G. Castex-P. Surer-G. Becker, 
alcune delle più note. Nessun dubbio sulla paternità della prima traduzione francese di Pamela hanno mostrato alcuni dei più recenti storici del romanzo francese del Settecento da Georges May ${ }^{59}$ ad Alain Montandon ${ }^{60}$; esattamente come avevano fatto, prima di loro, studiosi diversi ma sempre attendibili quali Daniel Mornet ${ }^{61}$, Charles Dédéyan $^{62}$, Jacques Bousquet ${ }^{63}$ e Chantal Thomas ${ }^{64}$ (anche qui per citare solo i primi che mi sono capitati sottomano); oppure un bibliografo esperto come S. Paul Jones ${ }^{65}$; e la lista potrebbe essere facilmente allungata ${ }^{66}$. Sulla medesima linea attributiva si situa infine la stragrande maggioranza degli studiosi italiani, in particolare quelli che più da vicino si sono occupati dei rapporti che la Pamela richardsoniana intrattenne con la cultura italiana: da Serafino Riva ${ }^{67}$ a Luciana Sciullo ${ }^{68}$, da Laura Crotti ${ }^{69}$ a Masolino d'Amico, autore dell'ultima accurata traduzione in italiano del capolavoro di Richardson ${ }^{70}$.

Tutto ciò nonostante che fin dal 1927 uno studioso americano, Frank Howard Wilcox, avesse dimostrato in maniera più che convincente che l'abbé Prévost non poteva essere stato e che, di fatto, non era stato il primo traduttore della Pamela richardsoniana ${ }^{71}$, e che anche prima di lui altri studiosi avessero emesso seri dubbi al riguardo. Fin dagli anni 70 dell'Ottocento, Jean-Barthélemy Hauréau si era, per esempio, fatto portavoce di una tradizione secondo cui il primo traduttore francese di Pamela sarebbe stato François-Alexandre Aubert de La Chesnaye-Desbois, uno dei tanti poligrafi del tempo, autore tra l'altro di alcune interessanti Lettres amusantes

Histoire de la littérature française, Paris Hachette, 1964, p. 38; M. CHARPENTIER et J. CHARPENTIER, Littérature. XVIII siècle, Paris, Nathan, 1987, p. 95.

(59) Cfr- G. MAY, Le dilemme du roman au XVIII siècle. Etude sur les rapports du roman et de la critique (1715-1761), New Haven, Yale University Press/Paris, Presses Universitaires de France, 1963, p. 166: «L'auteur anonyme d'une Lettre sur Pamela, parue peu après la publication de la traduction française de Pamela due à l'abbé Prévost [...]» e n. 1: «Pamela or Virtue rewarded (1740), traduit par Prévost sous le titre: Pamela ou la Vertu récompensée (1742); p. 270: «Richardson (Samuel), Pamela ou la Vertu récompensée (traduit par l'abbé Prévost de l'anglais: Pamela, or Virtue rewarded), Londres, Osborne, 2 vol., 1742».

(60) A. MonTANDON, Le roman au XVIII siècle en Europe, Paris, PUF, 1999, p. 241: «Le roman [Pamela] est très rapidement traduit en français par l'abbé Prévost en 1742-1743».

(61) Cfr. D. Mornet, Le Romantisme en France au XVIII' siècle, Paris, Hachette, 1925, p. 20.

(62) Cfr. C. DÉDEYAN, Jean-Jacques Rousseau et la sensibilité littéraire à la fin du XVIII siècle, Paris, Société d'Edution d'Enseignement Supérieur, 1966, p. 49.

(63) Cfr. J. Bousquet, Le dix-huitième siècle romantique, Paris, Jean-Jacques Pauvert, 1972, p. 131, n. 656.

(64) Cfr. Lettres de Madame du Deffand 1742 1780, Préface de Ch. Thomas, Paris, Mercure de France, 2002, p. 953 , n. 2.

(65) S.P. JONES, A List of French Prose Fiction from 1700 to 1750 , New York, The H.W. Wilson Company, 1939, p. XXVI: «Prévost's translation of Pamela was followed by Villarets's Anti-Pamela, ou Mémoires de M. D $D^{\text {*sek }}(1742) \gg$.

(66) Un esempio per tutti: tra gli studiosi che continuano ad attribuire a Prévost la traduzione di Pamela c'è Julie Candler Hayes che non solo ha attribuito senza alcuna esitazione la traduzione di Pamela all'abbé Prévost nel suo recentissimo libro Translation, Subjectivity and Culture in France and England 1600-1800 (Standford CA, Stanford University Press, 2008), ma ha anche inserito l'abbé Prévost, come autore della Préface che precede la traduzione di Pamela (data d'après l'edizione Liège, Bassompierre 1743), nella French Translators 16001800. A Online Anthology of Prefaces and Criticism (cfr. http://scholarworks.umass.edu/french_translators/80).

(67) S. Riva, Pamela a Venezia. Estratto dall'Annuario dell'Istituto Tecnico "Jacopo Riccati" di Treviso. Anno 1933-34, Treviso, Arti Grafiche Longo \& Zoppelli, 1935 , p. 3. Riva, che non cita le sue fonti ma che scrive sulla base di ricerche abbastanza accurate, avverte che «ad un anno di distanza dalla prima edizione inglese [Prévost] pubblicò in francese il romanzo di Richardson Pamela, or Virtue rewarded= Pamela ou la vertu récompensée» precisando però che «questa prima edizione francese era stata impressa: a Londres, chez T, Woodward 1741 ma l'anno dopo [...] ristampata sul continente colla data di Amsterdam».

(68) Cfr. L. Sciullo, Pamela da Richardson a Goldoni, «Quaderni di Lingue e Letterature», n. 1, 1976, p. 117.

(69) Cfr. C. Goldoni, Pamela fanciulla, Pamela maritata. A cura di Ilaria CRotTI, Venezia, Marsilio, 1995, p. 431: «Nota sulla fortuna».

(70) Cfr. S. RichardSON, Pamela o la virtù ricompensata. Traduzione di Masolino D'AMICO, Città di Castello, Frassinelli, 1995, Postfazione, pp. 629630.

(71) F.H. Wilcox, Prévost's translations of Richardson's novels, in «University of California $\mathrm{Pu}$ blications in Modern Philology», vol. 12, n. 5 (January 1927), pp. 341-411. 
et critiques sur les romans en général, anglois et français, tant anciens que modernes, una delle quali era stata dedicata proprio alla Pamela di Richardson ${ }^{72}$. Non è chiaro da dove J.-B. Hauréau abbia tratto questa informazione. La sua ipotesi fu, ad ogni modo, ripresa, alcuni decenni più tardi, da Joseph Texte, un attento studioso dei rapporti culturali intercorsi nel Settecento tra Francia ed Inghilterra, che di Aubert de La Chesnaye-Desbois fece però non il sostituto, bensì il collaboratore di Prévost, che rimaneva quindi, in qualche modo, il responsabile dell'operazione. Scriveva infatti Texte: «La traduction de Pamela paraît en 1742. Il semble que Prévost, absorbé en ce moment par d'autres travaux, se soit fait aider par un collaborateur» che lo studioso identificava per l'appunto in Aubert de La Chesnaye-Desbois ${ }^{73}$.

Con gli anni l'ipotesi, sostenuta inizialmente da J.-B. Hauréau, che Aubert de La Chesnaye-Desbois non fosse stato solo un collaboratore bensì il vero traduttore di Pamela, riprese però quota. Un ruolo importante in questo senso svolse, verso la metà del Novecento, Henri Roddier, che conosceva molto bene sia la vita di Prévost sia il lavoro con il quale Wilcox aveva cercato di smontare la tradizione che voleva Prévost autore della prima traduzione di Pamela. Roddier partì proprio da questa tradizione, di cui fece però subito notare le incongruenze (nessuno, dalla contemporanea $\mathrm{Ma}$ demoiselle Delaunay a Bernard d'Héry, il primo biografo di Prévost, passando per lo stesso Prévost, ha mai parlato del ruolo da lui avuto in questa traduzione), e fece propria l'osservazione, a suo avviso «essentielle», di Wilcox secondo cui il confronto tra le traduzioni di Clarissa e di Grandison, sicuramente di Prévost, e quella di Pamela evidenzia l'enorme divario che quest'ultima presenta con le abitudini traduttorie di Prévost, che allo studioso parve quindi del tutto estraneo alla traduzione francese di Pamela; tanto più che un attento esame della biografia lo portò ad escludere anche l'ipotesi, formulata da Texte, di una sua collaborazione con Aubert de La ChesnayeDesbois che, agli occhi di Roddier, apparve di conseguenza come l'unico (possibile) traduttore di Pamela. «Aubert de La Chesnaye - fece infatti notare Roddier a conclusione del suo ragionamento - profita d'un court séjour à Londres pour traduire $\mathrm{Pa}$ mela [... ] qui fut publiée en français dès janvier 1742, si l'on croit Wilcox. Comment Prévost aurait-il collaboré à l'ouvrage puisqu'il se trouvait encore à Francfort au début de novembre 1741 ? $\gg^{74}$.

Se la tesi della collaborazione non cadde del tutto (essa fu ripresa, per esempio, nel 1948 da Charles Alfred Rochedieu ${ }^{75}$ e, in anni più recenti, da P. M. Conlon ${ }^{76}$ e da Béatrice Didier ${ }^{77}$ ), quella secondo cui la paternità della prima traduzione francese di Pamela spetta al solo Aubert de La Chesnaye-Debois ha fatto un assai maggior numero di proseliti; e se non è, come abbiamo visto, riuscita a scalzare la tradizionale attribuzione a Prévost, è però stata fatta propria, in anni molto recenti, da studiosi

(72) J.-B. HAurÉAu, Histoire littéraire du Maine, Paris, Dumoulin,1870, t. I, p. 114. A proposito delle Lettres amusantes et critiques sur les romans, J.-B. Hauréau scrive tra l'altro: «On lit encore dans les Lettres amusantes tout un chapitre en l'honneur de la Pamela de Richardson. Or, La Chesnaye-Desbois passe pour avoir, le premier, traduit ce roman en Français: Pamela, ou la vertu récompensée».

(73) J. TEXTE, Jean-Jacques Rousseau et les origines du cosmopolitisme littéraire. Etudes sur les relations littéraires de la France et de l'Angleterre au XVIII siècle, Paris, Librairie Hachette et Cie, 1895 , p. 193.

(74) H. Roddier, L'abbé Prévoast. L’homme et l'œuvre, Paris, Hatier-Boivin, 1955, p. 167.

(75) C.A. RochedieU, Bibliography of French translations of English works, Chicago, The University of Chicago Press, [1948], ad vocem: «Pamela, ou la vertu récompenaée, ouvrage traduit de l'anglois [par l'abbé Prévost et Aubert de la Chesnaye des Bois], Londres, chez Jean [sic] Osborne, $1742 \gg$.

(76) P.M. Conlon, Le siècle des lumières. Bibliographie critique, t. IV (Genève, Librairie Droz, 1986), p. 515.

(77) B. Didier, Le siècle des Lumières, Paris, MA Editions, 1987, p. 344: «Pamela fut traduit en français par Aubert de la Chesnaye et par Prévost». 
avveduti quali Colin Duckworth ${ }^{78}$ e N.R. Johnson ${ }^{79}$, oltre che da noti bibliografi quali Alexandre Cioranescu e gli autori della Bibliographie du genre romanesque français 1751-1789 che, abbandonata la tesi sostenuta a suo tempo da Jones, hanno espresso la loro propensione per Aubert de La Chesnaye-Desbois ${ }^{80}$; senza dimenticare che lo stesso Wilcox, pur non prendendo una posizione netta al riguardo, aveva dato a suo tempo Aubert de La Chesnaye-Desbois come il possibile se non il probabile autore della prima traduzione francese di Pamela ${ }^{81}$. Altri, come gli autori dei Cataloghi della Biblioteca Nazionale di Parigi, e della British Library di Londra o Laurent Versini, il grande conoscitore del romanzo epistolare francese del Settecento, hanno invece preferito lasciare la questione nell'incertezza ${ }^{82}$.

In realtà, nessuna delle due attribuzioni regge ad un esame attento. L'attribuzione all'abbé Prévost è già stata, come abbiamo detto, confutata, e con validi motivi, da F. H. Wilcox e, più recentemente anche da Louis Thevenon; per cui ci limiteremo a ricordare brevemente le considerazioni che hanno portato questi due studiosi a rifiutare all'autore di Manon Lescaut la paternità della prima traduzione francese di Pamela. Dopo aver ricordato che nessuna testimonianza coeva ha mai attribuito la traduzione francese di Pamela a Prévost e che egli stesso non ha mai menzionato questa traduzione tra le sue opere, Wilcox ha fatto notare come il metodo usato per tradurre Pamela sia completamente diverso da quello che Prévost ha utilizzato per tradurre Clarissa e Grandison: la prima è una traduzione sostanzialmente fedele al testo inglese ${ }^{83}$ mentre la seconda e soprattutto la terza sono degli adattamenti sempre più liberi e sempre più sensibili al gusto dei lettori (francesi) cui essi erano diretti. Wilcox concludeva il suo esame esprimendo la sua preferenza per Aubert de La Chesnaye-Desbois che, a suo avviso, possedeva tutte le qualità per svolgere bene quel lavoro: «He was the kind of hack writer who would have been likely to do such work, and his Lettres amusantes et critiques sur les romans indicate that he knew English ${ }^{84}$. Nella sua thèse consacrata all'Étude de la sensibilité dans la traduction française de "Pamela" de Richadson, Louis Thevenon non si è occupato del problema della sua paternità in maniera specifica. L'attenta analisi alla quale egli ha sottoposto la traduzione di Pamela e il confronto da lui istituito con le teorie sulla traduzione espresse da Prévost nelle «Préfaces» alle sue traduzioni di Clarissa e di Grandison (e che Thevenon ha confrontate con quelle dei suoi contemporanei, o di coloro che in questo lavoro lo avevano preceduto), ha tuttavia portato anche lo studioso francese ad escludere l'abbé Prévost dal novero dei possibili traduttori di Pamela; ed a optare per una ipotesi sulla quale avremo modo di ritornare più avanti ${ }^{85}$.

(78) C. Duckworth, Madame Denis's unpublished "Pamela". A link between Richardson, Goldoni and Voltaire, «Studies on Voltaire and the Eighteenth Century», vol. 76 (1970), pp. 37-53.

(79) Cfr. Correspondance de Madame Graffigny, t. cit., p. 319 .

(80) Cfr. A. Cioranescu, Bibliographie de la littérature française du XVIII siècle, Paris, Editions du Centre National de la Recherche Scientifique, 1969-1970, t. II, alla voce «Prévost» e A. MARTin, V. Mylne, R. Frautschi, Bibliographie du genre romanesque français 1751-1800, London, Mansell / Paris, France Expansion, 1977, p. 135.

(81) F.H. Wilcox, op. cit., p. 352: «Aubert de la Chesnate-Desbois may well have been the translator».

(82) Nel Catalogue della Biblioteca Nazionale di Parigi, Paris, t. 000, p. 287, alla «voce»: «Pamela, ou la vertu récompensée. Traduit de l'anglais [de S. Richardson]. Londres, J. Osborne, 1742. 4 parties en 2 vol.in-12», si legge: «Cette traduction est attribuée à l'abbé A.-F. Prévost, et aussi à Aubert de La Chesnaye». Nell'Integrated Catalogue della British Library, a commento dell'item «Pamela, ou la vertu recompensée. Traduit de l'Anglois. A Amsterdam, aux dépen de la Compagnie, 1742», dopo l'indicazione «Traduit de l'Anglois» c'è l'annotazione: «by François A. Aubert de la Chesnaye des Bois? The translaton is sometimes attributed to Antoine F. Prévost d'Exiles».

L. Versini, Le roman épistolaire, Paris, PUF, 1979, p. 71: «Pamela (1741), traduit en français dès 1742 par l'abbé Prévost ou Aubert de La Chesnaye des Bois».

(83) «The translator of Pamela translated the novel in its entirety, omitting nothing of importance, even from the phenomenally dull continuation» (WILCOX, op. cit., p. 352).

(84) Ibid.

(85) L. Thevenon, Étude de la sensibilité dans 
Per quanto riguarda Aubert de La Chesnaye-Desbois, non siamo ancora riusciti a capire quando e come sia nata l'idea che egli abbia preso parte, o sia addirittura stato l'autore della prima traduzione francese di Pamela, così come ha attestato J.-B. Hauréau a metà dell'Ottocento, e come ha ribadito all'inizio del Novecento Paul Dottin, sulla base peraltro di una lettura un po' strana delle Lettres amusantes et critiques sur les romans dello stesso Aubert de La Chesnaye-Desbois ${ }^{86}$. Basta infatti leggere con un po' di attenzione proprio la terza delle succitate Lettres, quella consacrata per l'appunto a Pamela, per rendersi conto che Aubert de La ChesnayeDesbois può assai difficilmente essere considerato l'autore della prima traduzione francese del romanzo di Richardson. Aubert de La Chesnaye-Desbois inizia dando conto dell'incertezza nella quale i primi lettori, e lui per primo, si sono trovati circa la natura e l'origine stessa dell'opera. «Selon quelques-uns ce sont des Lettres monotones, qui composées avec tout le froid qu'on reproche à vos insulaires - fa notare alla sua supposta interlocutrice inglese -, ont pris naissance sur les bords de la Seine, $\&$ ne doivent leur origine qu'à quelque Anglico François, qui pour avoir voulu copier les mœurs de votre Nation, en a fait un ridicule assortiment. Selon d'autres, c'est la traduction d'une Histoire écrite \& arrivée il y a trente ans sur les bords de la Tamise», mentre per un certo «Jacobite, qui depuis bien des années fait son séjour en France», Pamela è «un Etre de Raison, sorti du cerveau imaginaire d'un de vos Philosophes de Londres»; ritenendo, quant à lui, che «On attribue à tort l'Ouvrage à quelque Auteur de Paris» perché «tout mauvais écrivain qu'il seroit, il n'auroit pu s'empêcher d'y glisser de nos maniéres françoises» ${ }^{87}$. Aubert de La Chesnaye-Desbois passa poi a confrontare le intenzioni dell'autore di Pamela, così come sono state espresse nella «Préface de l'Editeur», con quello che l'opera effettivamente propone e offre. Ne risulta una critica sistematica, e tutto sommato molto dura, del romanzo di Richardson che non solo non mantiene le promesse formulate dall'Editeur, ma che risulta anzi profondamente deludente e tale da giustificare ampiamente l'atteggiamento dei Francesi i quali, dopo un entusiasmo iniziale, nato soprattutto dalla curiosità, se ne sono ben presto allontanati. «Les personnes qui ont le plus de goût pour ces sortes d'Ouvrages - fa notare Aubert de La Chesnaye-Desbois - l'ont acheté, comme ils achetent tous les autres Romans, à dessein de s'en amuser, mais presque toutes se sont ennuyées en le lisant», tanto che molte di esse «n'ont fait que le parcourir». A conferma e a spiegazione di questo atteggiamento, e delle ragioni che lo giustificano, il critico porta la testimonianza, da un lato, di un lettore di professione, dall'altro del lettorato femminile, notoriamente numeroso ed interessato: «Un célébre Académicien, juge équitable de tous les différens Ouvrages d'esprit [...] a blamé l'Auteur de sa trop grande prolixité \& de ses négligences. Nos dames sentant dès le premier Volume l'ennui que ces Lettres inspirent, ne les ont luës qu'en bâillant», per concludere che, contrariamente a quanto aveva enunciato l'Editeur, «plaire \& divertir n'est point ce que fait Pamela, puisque j'apprends du Public [...] qu'il a fort ennuyé» ${ }^{88}$.

Eguale divario tra intenzioni dichiarate e risultati raggiunti il critico rileva circa l'effetto morale prodotto dall'opera. Mentre l'Editeur aveva dichiarato che lo scopo precipuo che deve porsi un romanzo, e che Pamela in particolare si è posto, è quello di «instruire E cultiver l'esprit E le cour des jeunes gens, de l'un E de l'autre sexe»,

la traduction française de "Pamela" de Richardson. Thèse présentée à l'Université de Grenoble le 30 novembre 1977, pp. 217-218.

(86) P. DotTin, op. cit., p. 117.

(87) Aubert de La Chesnaye-Desbois, Lettres amusantes et critiques sur les Romasn en général
Anglois et François, tant anciens que modernes. Adressées à Miledy $W^{* *}$ Première Partie, A Paris, Gissy, Bordelet \& David, M.DCC. XLII, pp. 44-45. (88) Ibid., p. 47, I corsivi, qui come altrove, sono nel testo. 
«Cet Ouvrage - fa notare il critico - n'instruit, \& ne cultive pas mieux l'esprit \& le cœur des jeunes gens de l'un \& de l'autre sexe» di quanto non li abbia «divertis» ${ }^{89}$.

Je vois dans Pamela - spiega - une petite personne, qui n'a pas la simplicité que l'Auteur lui prête. En effet, une fille qui a beaucoup lu, qui cite dans ses Lettres des fragmens de l'Histoire, \& de la fable, qui raisonne en Phisionomiste sur le caractere des trois Dames, qui rendent visite à son Maître, qui rit sous cape, quand on la louë sur sa beauté, n'est-elle qu'une simple fille? [...] Aimant les douceurs qu'on lui compte [sic], se donnant le plaisir d'en faire part à ses parens, extreme dans ses éloges, emportée dans ses invectives, curieuse dans ses ajustemens, n'étant pas toujours fâchée des poursuites du Milord, peut-être n'affectant une vertu sauvage, que pour mieux arriver à son but, elle n'a pas cette belle simplicité qu'on lui donne ${ }^{90}$.

Per cui, se Pamela istruisce e serve da modello, non è certo per le giovani che in lei dovrebbero scorgere un modello di virtù, bensì per le «filles de son espèce» che da lei e dal suo esempio possono imparare «à bien menager la passion que des hommes au-dessus de leur état peuvent concevoir pour elles».

Ma risultati soddisfacenti, e consoni con quanto promesso, Pamela non è riuscita a raggiungere neppure nel modo in cui i vari personaggi sono stati delineati. La quinta «maxime» formulata dall'Editeur delle Lettres de Pamela, ricorda Aubert de La Chesnaye-Desbois, afferma che «Dans un Roman on doit trouver des caractères vrais \& bien soutenus» ${ }^{91}$. Ora, precisa il critico, «ceux qui sont tracés dans Pamela, ne m'ont pas paru tels». Tralasciando di soffermarsi su quelli dei personaggi minori, come Jonathan e Longman per esempio, i quali «ne sont pas des personnages assez intéressans pour chercher à vous débrouiller leurs caracteres», Aubert de La Chesnaye-Desbois limita la sua analisi sui maggiori, per osservare che «Celui de Pamela est assez difficile à connoître. La ruse, l'artifice y entre pour quelque chose. Une vertu, mais je ne sçais quelle vertu de temperamment, la fait mal combattre, \& pour la faire trop long-tems combattre, son caractère n'est ni vrai ni proprement soutenu», e che «Celui du Milord n'est pas non plus avantageux»; in quanto «ce seigneur Anglois manque à l'éducation qu'il a reçue, en n'ayant dans la bouche que des expressions basses», non riuscendo a celare la sua perplessità di fronte al comportamento, a dir poco inconguente, che Mr. B*** ha con Pamela:

En Angleterre, Madame, j'entends parmi le monde poli, les Amans traitent-ils leurs Maitresses de sottes, de salopes, e\&? Si le caractere de Mylord est tracé d'après nature, mais je n'en crois rien, il faut avouer que dans la Grande Bretagne on fait grossierement l'amour. Comme il faut que Pamela triomphe, je sens que le caractere de son Maitre ne doit pas être des plus brillans. C'est un jeune homme, qui ne sçachant pas la belle manière d'attaquer un cœur, est comme un Ecolier devant Pamela, qui se défendant en Dévote, le mene plus loin qu'il ne pense, $\&$ il doit être honteux pour lui de n'avoir pas connu d'abord que par les menaces \& la violence, on ne vient presque jamais à bout de rien ${ }^{92}$.

Aubert de La Chesnaye-Desbois raccoglie insomma e fa proprie le accuse che, sia dal punto di vista morale che dal punto di vista estetico, una parte della critica francese aveva o avrebbe espresso nei confronti dell'opera di Richardson. È difficile, in questa situazione, pensare che Aubert de La Chesnaye-Desbois sia stato in qualche modo coinvolto nella traduzione francese di Pamela; ancora di più che, come hanno supposto P. Dottin e H. Roddier, egli sia stato invitato dall'agente letterario di Ri- 
chardson a Londra per lavorare fianco a fianco con l'autore dell'opera e sotto la sua stretta sorveglianza.

Al traduttore di Pamela Aubert de La Chesnaye-Desbois ha del resto fatto allusione più volte nel corso della sua Lettre. All'inizio egli rimprovera al Traducteur di aver posto Pamela sullo stesso piano del Cid di Corneille, in quanto vittima, come l'opera francese, di critiche tanto aspre quanto, a suo avviso, ingiuste. «Quelle difference aussi du Cid à Pamela! L'un est-il à comparer à l'autre?» commenta però il critico, che prosegue non nascondendo il suo stupore per il toupet di chi ha osato mettere allo stesso livello un «Roman fort uniment écrit [et] rempli d'ennuieuses fadaises» ed una «Tragédie qui sera toujours l'admiration des Connoisseurs», per concludere: «Il seroit glorieux pour l'Auteur du Roman Anglois de se voir égalé au célébre Auteur du Cid. Mais le Traducteur me paroit un peu trop prévenu en faveur de l'Ouvrage qu'il vient de nous traduire» ${ }^{93}$.

Più avanti, commentando l'elogio formulato su Pamela dall'autore della Lettera indirizzata «A l'Editeur du Livre intitulé Pamela, ou la vertu recompensée» da J.B.D.F. il quale aveva sottolineato in particolare «l'agréable simplicité du style, la clarté \& la justesse de ses expressions» fino a fare di Pamela «un modèle dans son genre», Aubert de La Chesnaye-Desbois chiede alla supposta desinataria inglese della sua Lettre di quale modello possa mai trattarsi in quanto, fa osservare, se Pamela è destinata a servire da modello «à vos Ecrivains Anglois» è giocoforza riconoscere che «l'Ouvrage a beaucoup perdu d'être traduit». Ma, si chiede, «pourroit-il être défiguré jusqu'au point, qu'on nous l'eût rendu ridicule?» per precisare subito dopo che «Un Traducteur, quelque médiocre qu'il soit, ne peut rien changer au fond de l'Ouvrage», e che «s'il n'est pas capable d'en rendre toutes les beautés, du moins en fait-il paroittre quelques-unes, qui font juger des autres» ${ }^{94}$. Sono osservazioni, l'una e l'altra, che Aubert de La Chesnaye-Desbois si sarebbe ben guardato, ci sembra, dal formulare se fosse stato in qualche modo implicato nella traduzione dell'opera; a maggior ragione se ne fosse stato egli stesso il traduttore ${ }^{95}$; per cui risulta praticamente impossibile che Aubert de La Chesnaie-Desbois possa avere, in qualche modo, collaborato alla prima traduzione francese di Pamela.

Scartati i due principali indiziati, è giocoforza seguire altre piste, incamminarsi su altre strade. Prima di partire, è tuttavia opportuno interrogare i contemporanei, prendere in considerazione i giudizi, o almeno le impressioni che sul problema espressero i lettori dell'epoca. La prima testimonianza al riguardo è offerta da Mme de Graffigny che, in una lettera del 20 giugno 1742 all'amico Antoine Devaux presenta, come abbiamo già visto, la Pamela francese come «un Roman anglois traduit par un Anglois» ${ }^{96}$. Una testimonianza quasi identica ha lasciato Mme de Staal-Delaunay che in una lettera del giorno dopo a M. D'Héricourt definisce Pamela «un Roman Anglois traduit en françois par un Anglois $»^{97}$. Sono due testimonianze molto importanti le quali, assai più che il risultato di un'improbabile informazione, rappresentano la reazione che le due raffinate lettrici hanno avuto di fronte ad un testo non del tutto in linea con i canoni del gusto francese, e che perciò stesso non poteva provenire che da una persona la quale della lingua di Voltaire non aveva una conoscenza ed una padronanza perfette; un inglese appunto.

Molto simile nella sostanza, anche se meno precisa nella forma, è la testimonianza lasciata da Crébillon fils che in una lettera del mese seguente a Lord Chesterfield,

(93) Ibid., pp. 45-46.

(94) Ibid., p. 46.

(95) A queste conclusioni era già giunto, sostanzialmente, anche A.- D. McKillop, op. cit., p. 93.
(96) Correspondance de Madame de Graffigny, p. 318.

(97) Lettres de Mlle De Launay cit., p. 219. 
dopo aver evocato il grandissimo successo che l'opera di Richardson aveva incontrato a Parigi, confessava di avere anche lui ceduto alla tentazione, traendone peraltro un'impressione molto positiva:

Je l'ai enfin lue à mon tour; et n'en déplaise à nos caillettes et à nos petits maitres caustiques qui ne jugent que par ce qui est bien écrit, sans pouvoir se connoître en stile, et qui réduisent tout au jargon de leurs ruelles, je l'ai trouvée fort interessante. J'aurois souhaité, à la vérité, que le traducteur se fût un peu plus élevé. On trouve dans l'ouvrage beaucoup de choses basses, qui me paroissent être moins de l'ouvrage que de la traduction. Une chose qui m'en plaît, quoiqu'elle déplaise à beaucoup de gens, c'est qu'elle a conservé à l'original ses mœurs, et ne lui a pas impertinemment substitué les nôtres. Pamela, habillée à la française, auroit, je crois, été bien ridicule ${ }^{98}$.

Riserve, più o meno forti, sulla qualità della traduzione espressero anche alcuni lettori diciamo così professionali dell'epoca: l'autore della Lettre sur Pamela, ad esempio, definisce la Pamela francese «une mauvaise traduction d'un Original singulier» ${ }^{99}$, mentre per la Bibliothèque françoise «Le stile dont on s'est servi dans la traduction de cet Ouvrage est simple \& touchant: Mais il n'est ni pur ni exact» precisando che «Le traducteur paroît ignorer non seulement les termes du bel usage, mais le génie de notre Langue» ${ }^{100}$. Anche all'autore della Lettre à Monsieur l'Abbé Desfontaines sur Pamela lo stile dell'opera, tanto elogiato da Desfontaines, è parso «plein de solécismes, barbare, en un mot, le stile d'un homme qui ne connoît point du tout le génie de la Langue Françoise» ${ }^{101}$. Al di là delle differenze che le caratterizzano, tutte queste indicazioni convergono, mi sembra, su un punto, che va a confermare le impressioni formulate a caldo dalle due lettrici che abbiamo evocato all'inizio di questa breve rassegna: la prima traduzione francese di Pamela non è stata fatta né da un Francese e neppure in Francia, bensì in Inghilterra e da uno uomo che della lingua di Voltaire aveva una conoscenza e/o una padronanza buona ma non perfetta.

I dati esterni di cui disponiamo confermano del resto almeno la seconda parte della conclusione: la prima edizione francese di Pamela uscì in effetti, come abbiamo visto, a Londra alla fine del 1741 in due tomi, il primo dei quali con l'indicazione «A Londres, chez Thomas Woodward, au Croissant entre les Portes du Temple; Et Jean Osborn, à la Boule d'Or, dans Pater Noster Row, près S. Paul», il secondo con l'indicazione del solo «Jean Osborn» che, come abbiamo cercato di dimostrare nella prima parte di questo lavoro, era nel frattempo subentrato interamente al socio nell'affaire della traduzione, ma soprattutto della commercializzazione della traduzione francese di Pamela; la quale, concepita inizialmente dai due editori «for the use of Schools», quindi con ambizioni abbastanza modeste e per un pubblico essenzialmente insulare, scavalcò ben presto la Manica e fu non solo venduta ma addirittura stampata anche a Parigi (molto verosimilmente, con l'accordo di John Osborn), dopo una rapida revisione dello stile che, tutto sommato, rimase però, come abbiamo visto, quello originale, prima di esserlo anche in Olanda.

A chi fu affidato o chi si assunse il compito di tradurre in francese (nei limiti e con le finalità che abbiamo dianzi ricordate) il primo romanzo di Richardson? Qui, gli indizi di cui disponiamo si fanno più labili e le ipotesi emesse negli anni non sono

(98) C. CréBILlon, Euvres complètes. Edition critique sous la direction de Jean SGARD, t. IV, Paris, Multimedia, 2002, p. 830. Lettera del 26 luglio 1742 .

(99) Lettre sur Pamela, Londres, s.e. 1742, p. 3.
(100) Bibliothèque françoise, ou Histoire littéraire de la France, t. XXXV (seconde Partie). A Amsterdam, chez H, De Hondt, M.DCC.XLII, p. 323.

(101) Lettre à Monsieur l'Abbé Desfontaines, sur Pamela, M.DCC. XLII, pp. 18-19. 
sempre state, mi sembra, sufficientemente motivate. P. Dottin nei primi decenni del Novecento aveva supposto che Richardson, geloso della sua opera, avesse chiesto al suo agente letterario, Jean-Baptiste de Fréval, di «trouver un traducteur habile et expéditif, qui fût capable de préparer une Pamela française avant que les pirates eussent pu s'en emparer» e che il solerte agente avesse per questo «recruté toute une équipe de traducteurs qui s'attela à la traduction du roman» e di cui facevano parte «peut-être l'abbé Prévost, certainement le mathématicien Clairaut et Aubert de la Chesnaye-Desbois qui, dans ses fameuses Lettres sur les romans, devait faire à Pamela une réclame trop habile pour être tout à fait désintéressé» ${ }^{102}$. P. Dottin, che non cita le sue fonti, non dice né su cosa abbia basato la sua ricostruzione né da dove provengano i nomi da lui fatti; egli ha tuttavia il merito di avere intuito che la traduzione si fece a Londra, e non a Parigi, e che i traduttori (e non il traduttore) lavorarono molto probabilmente in équipe, e in contatto, se non sotto il controllo dell'autore, che fornì loro delle correzioni e del materiale inedito con cui impreziosire la traduzione. Paul Dottin capì anche che da un lavoro fatto da una équipe, non poteva uscire che una traduzione «très fidèle pour l'époque» in quanto «chaque équipier, ignorant la mentalité de son voisin, rendit en français le texte intégral» ${ }^{103}$. Seppure con le persone sbagliate, come abbiamo visto, P. Dottin andò probabilmente assai più vicino a ciò che effettivamente successe di quanto non si sarebbe inizialmente potuto supporre sulla base di indicazioni non suffragate da dati oggettivamente riscontrabili: non solo collocò la traduzione in terra inglese, ma la fece opera di «manœuvres littéraires» piuttosto che di scrittori già famosi, come poteva essere Prévost ma, in fondo, anche Aubert de La Chesnaye-Desbois.

Le analisi condotte recentemente da due giovani studiosi sulla traduzione francese di Pamela sembrano confermare questa ipotesi. Louis Thevenon ha distinto almeno due traduttori, facendo presente che quello che ha tradotto i testi in versi non è lo stesso che ha tradotto la parte in prosa ${ }^{104}$. Ad una conclusione molto simile è pervenuta, in anni più recenti, Maria Gabiella Zerman la quale ha creduto di poter individuare addirittura tre traduttori: uno per ciascuno dei due volumi dell'edizione inglese, le cui traduzioni paiono riflettere pratiche leggermente diverse, ed uno per i componimenti in versi, la cui traduzione obbedisce ad un'altra logica ${ }^{105}$. Anche un'altra osservazione di Louis Thevenon sembra poter confermare questa ipotesi. Dopo aver ricordato che la pratica traduttoria dei Francesi obbediva, seppur in gradi e forme diverse, alla necessità di adeguare lo stile dell'opera tradotta al gusto francese, il che comportava spesso trasformazioni anche profonde al testo di partenza, Thevenon ha fatto notare che nell'ambito del Rifugio, dove il lavoro di traduzione rispondeva ad una logica più utilitaristica, queste preoccupazioni erano meno forti e che, di conseguenza, le traduzioni operate in quel contesto erano più rispettose del testo originale, come è, per l'appunto, il caso della prima traduzione francese di Pamela. È del resto quanto appare dalla «Préface» che apre la traduzione:

Disons un mot de notre traduction. Nous avons tâché de la rendre aussi fidelle qu'il nous a été possibile vu la différence des Langues. On sait que la langue Angloise n'est pas tout aussi châtiée que la Françoise. On souffre dans celle-là des Expressions qu'on ne permettroit pas dans celle-ci [..] C'est ce qui nous a obligez à rendre le sens de notre Auteur, plutôt que de suivre exactement ses Expressions ${ }^{106}$.

(102) P. DotTin, op. cit, p. 131.

(103) Ibid.

(104) L. Thevenon, op. cit., p. 217.

(105) M. G. Zerman, La "Pamela" di Richardson e la sua prima traduzione francese, Relatore: Prof. Franco Piva, Università degli Studi di Verona, a.a. 2001-2002, pp. 227-228.

(106) Pamela cit., «Préface», p. n.n. 
La «Préface» è interessante anche per un altro motivo; in quanto sembra confermare la presenza al tavolo della traduzione di più soggetti. Il plurale che vi viene usato non pare infatti nè un plurale di cortesia né un plurale majestatis.

Alcuni anni più tardi, McKillop fece propria l'idea di Dottin, precisandola però nei dettagli. Esclusi, per motivi sia pratici sia teorici, Prévost e Aubert de La Chesnaye-Desbois, il critico inglese puntò la sua attenzione sulla colonia francese di Londra. Trovata nelle Anecdotes raccolte da Nichols l'indicazione secondo la quale i versi presenti in Pamela erano stati tradotti da César de Missy, «a clergyman attached to the French Chapel of the Savoy», era logico pensare che anche il resto dell'opera fosse stato tradotto «by some Frenchman in London» ${ }^{107}$, anche se lo studioso inglese non seppe andare oltre ed essere più preciso. Era ad ogni modo, ormai chiaro che era nel contesto del Rifugio, o quantomeno della presenza francese nella capitale inglese, che occorreva cercare, per tentare di dare un nome al o ai responsabili della prima traduzione francese di Pamela. Un documento scoperto alcuni anni orsono da Jean Sgard ha proposto una soluzione che, all'apparenza, sembrava perfettamente compatibile con il contesto che è venuto via via delineandosi. In una lettera del 24 dicembre 1743 a Pierre Desmaizeaux, un protestante francese emigrato in Inghilterra, Charles de La Motte, personaggio di solito bene informato, lasciava intravedere non solo che la traduzione di Pamela era stata un lavoro eseguito in équipe nell'ambito del Rifugio londinese, ma dava anche delle indicazioni su alcuni membri di questa équipe:

M. Coste m'écrivit il y a quelques mois, que M. Bernard était l'auteur du Pamela qui a fait tant de bruit, Cependant aucun de ceux à qui j'ai dit cette nouvelle n'en veulent rien croire. Les uns prétendent que l'Auteur est un seigneur bien connu, d'autres que c'est l'imprimeur du Parlement. Qu'en dites-vous? On a dit avant cela que M. Bernard a traduit les deux premiers volumes, dont la traduction est imprimée à Londres, et que $\mathrm{M}$. de Bissy a traduit en vers les vers qui s'y trouvent. Les tomes III et IV ont été traduits par un Ministre Suisse, qui demeure ici chez M. Hop. Il est gouverneur de son fils. Il se nomme Bernand ${ }^{108}$.

Jean Sgard ha creduto di vedere nel Bernard di cui parla la lettera di Charles de La Motte, e che il contesto dice nipote di Barbeyrac, Jean-Frédéric Bernard «journaliste, éditeur et traducteur fort connu» e, seppure con minore sicurezza, nel M. de Bissy che avrebbe tradotto i versi, Jean Rousset de Missy ${ }^{109}$. Ma né Jean-Frédéric Bernard, né Jean Rousset de Missy appartengono a quel Rifugio londinese all'interno del quale, come abbiamo visto, sembra essere nata ed essersi realizzata l'idea di tradurre in francese il romanzo di Richardson. La lettura che di quella lettera ha dato Sgard, secondo cui «en supposant [...] que la traduction de Pamela fut l'œuvre d'une équipe de réfugiés protestants, peut-être sous la direction de Jean-Frédéric Bernard, on a des chances d'être dans le vrai», era esatta solo parzialmente. Se équipe, come pare, ci fu, essa non fu certamente diretta da Jean-Frédéric Bernard, che abitualmente risiedeva ed operava ad Amsterdam, non a Londra. Le indicazioni contenute nella lettera di Charles de La Motte si riferiscono con ogni probabilità, vista anche la data della lettera, alla revisione alla quale la traduzione "inglese" di Pamela fu sottoposta, nel corso del 1742, prima di essere presentata dalla compagnia dei Libraires Associés di Amsterdam, in un'edizione destinata a fare concorrenza a quelle che nel frattempo erano apparse a Parigi, ed a cui si sarebbe aggiunta subito dopo, anche la traduzione dei tomi III e IV dell'opera, alla quale la lettera del La Motte faceva pure allusione.

(107) Mc Killop, op. cit., p. 94.

(108) La Lettera, citata da J. SGARD, Prévost romancier, Paris, Corti, 1968 , pp. 539-540, è conser- vata alla British Library sotto la segnatura : Add. mss. 4287, f ${ }^{\circ} .170$.

(109) Ibid., p. 540, n. 27. 
La conferma parrebbe venire da una pagina della Bibliothèque britannique per i mesi di gennaio, febbraio e marzo 1744 . Dopo aver presentato la traduzione francese di Anti-Pamela, la parodia della Pamela richardsoniana che, attribuita tradizionalmente ad Elyza Haywood, era stata tradotta da Eléazar Mauivllon, l'autore faceva notare, come abbiamo già visto, che «les traductions de ces deux Romans de Pamela, \& d'Anti-Pamela, ne sont nullement bien écrites e che la prima in particolare [quella di Pamela], era talmente «irreguliere dans l'édition de Londres», che «on a été obligé de [la] rajuster tant bien que mal en beaucoup d'endroits des Editions d'Amsterdam $\gg^{110}$. In realtà, la soluzione era, ed, è forse più vicina, e più semplice, di quanto si potesse supporre. Dato ormai per acquisito che la prima traduzione francese di Pamela è stata concepita ed eseguita a Londra, perché non pensare, sulla scia di quanto ha in fondo suggerito Paul Dottin il quale, pur non declinando mai le sue fonti, sembra disporre di informazioni sconosciute ad altri, ad una équipe costituitasi attorno a quel Jean-Baptiste de Fréval che abbiamo visto molto vicino a Richardson, e della quale lo stesso de Fréval sarebbe stato non solo il coordinatore, come ha supposto Dottin, ma l'elemento portante? Chi più e meglio di lui, poteva ottenere da Richardson $\mathrm{i}$ suggerimenti, le correzioni ed i materiali inediti che lo scrittore inglese mise a disposizione di coloro che tradussero in francese la sua Pamela? È solo un'ipotesi; alla quale hanno pensato tuttavia anche gli editori della "Pamela" Controversy che recentemente hanno presentato la traduzione francese di Pamela come «undertaken with Samuel Richardson's approval, probably by Jean-Baptiste de Fréval with verse translation by César de Missy» ${ }^{111}$. Il nome del traduttore della parte in versi proviene, come abbiamo detto, da John Nichols che in una pagina delle sue Biographical and Literary Anecdotes of William Bowyer (lo stampatore della Pamela francese!) fa notare che «the four poetical pieces in the French Translation of Pamela» erano opera di un certo César de Missy, che McKillop ha, come abbiamo visto, definito «a clergyman attached to rhe French Chapel of the Savoy» e che anche altre fonti danno presente a Londra negli anni in cui la traduzione di Pamela fu portata a termine ${ }^{112}$. Il cerchio sembra via via restringersi ed il ruolo dell'enigmatico Jean-Baptiste de Fréval assumere connotazioni sempre più precise ed importanti. Non sappiamo se della traduzione di Pamela egli si occupò da solo o se, come è probabile, si fece aiutare da qualche altro, non solo per la parte in versi ma anche per quella in prosa. Le informazioni di cui disponiamo sono ancora troppo frammentarie e labili, per fornire una risposta più precisa, anche se le indicazioni provenienti dalla Corrispondenza di Richardson raccolta agli inizi dell'Ottocento da Anna Laetitia Barbauld, e che indicano Clairaut tra i traduttori di Pamela, sembrano andare nella direzione da noi ipotizzata ${ }^{113}$.

Che nella traduzione di Pamela Fréval abbia avuto un ruolo importante se non preponderante, e certamente più significativo di quanto si sia fino ad ora ipotizzato, pare del resto confermato da due indicazioni fino ad ora ignorate o passate sotto silenzio. La prima è contenuta in Pamela censured, una sorta di pamphlet lanciato alla fine di aprile del 1741 contro l'opera di Richardson. Nelle pagine iniziali l'autore (per noi anonimo ma assai bene informato di quanto capitava attorno a lui, e soprattutto nel mondo delle Lettere) non solo dava conto della prossima traduzione francese di Pamela ma lasciava anche chiaramente intendere che in questa operazione un ruo-

(110) Bibliothèque britannique, cit., t. XXII, art. VII, p. 365.

(111) The "Pamela" Controversy cit., t. I, p. xxv.

(112) Ad esempio, la «notice» dedicata a César de Missy da John Patrik Lee nel Dictionnaire des journalistes, Oxford, Voltaire Foundation, 1999, pp. $580-581$.

(113) The Correspondence of Samuel Richardson, selected from original manuscripts ... by Anna Laetitia Barbauld, London, R. Philips, 1804, t. I, pp. CCII. 
lo fondamentale lo aveva proprio Jean-Baptiste de Fréval, che l'autore non nomina esplicitamente ma che conosce meglio di quanto non voglia lasciare intendere:

The first of them insinuates a French Translation, and as I see one is since advertised to be published, if may not be amiss to congratulate the Gentleman, whoever he is, on the lucky Thought, and with him as much Succes on his beeing Translator, as you have met with in beeing Editor» ${ }^{114}$

L'altra indicazione, altrettanto interessante anche se meno diretta, proviene dalla Corrispondenza stessa di Richardson. Louis Thevenon ha fatto notare che in una delle sue lettere Richardson invitava il suo agente letterario a non proseguire nella traduzione di Clarissa in quanto essa era già stata intrapresa da un Francese (l'abbé Prévost $)^{115}$. Se aveva iniziato a tradurre Clarissa, è, mi pare, molto probabile, se non sicuro, che la stessa cosa egli avesse fatto anche con Pamela. Non è forse inutile ricordare che de Fréval non era nuovo ad operazioni di questo tipo. Come hanno fatto osservare T. C. Duncan Eaves e Ben D. Kimpel, «De Fréval was a minor French author and translator then living in London» ${ }^{116}$.

Si tratta, ovviamente, solo di un'ipotesi, che ha bisogno di ulteriori verifiche e di più precise conferme; verso la quale sembrano tuttavia convergere tutta una serie di indizi, e che perciò stesso merita, mi pare, di essere tenuta nella più attenta considerazione.

FRANCO PIVA

(114) Pamela Censured; in a Letter to the Editor [...] London, Printed for J. Roberts, at the Oxford Arms, in Warwick-Lane, MDCCXLI, p. 14. «The first» indica l'autore della Lettera «A l'Editeur du Livre intitulé Pamela, ou la vertu récompensée» che, sulla base anche della sigla posta in calce alla Lettre, è stato individuato in Jean-Baptiste de Fréval. Visto dai più nel ruolo di agente letterario di Richardson, egli sembra avere svolto, in realtà, accanto allo scrittore inglese, un ruolo spesso più importante, equiparabile a quello del consulente letterario.

(115) L. ThevenOn, op. cit., p. 219.

(116) Cfr. Pamela by Samuel Richardson. Edited by T.C. Duncan Eaves and Ben D. Kimpel, Boston Houghton Mifflin Company, 1971, p. 4. L'anno prima della traduzione di Pamela, J.-B. de Fréval aveva infatti tradotto in inglese l'Histoire du Ciel dell'abbé Pluche (cfr. T. Kreimer \& P. Sabor, The "Pamela" Controversy cit., t. I, p. XLV). 